TITLE:

\title{
Models of the LHC diphoton excesses valid up to the Planck scale
}

\section{$\operatorname{AUTHOR}(\mathrm{S}):$}

Hamada, Yuta; Kawai, Hikaru; Kawana, Kiyoharu; Tsumura, Koji

\section{CITATION:}

Hamada, Yuta ... [et al]. Models of the LHC diphoton excesses valid up to the Planck scale. Physical Review D - Particles, Fields, Gravitation and Cosmology 2016, 94(1): 014007.

ISSUE DATE:

2016-07-08

URL:

http://hdl.handle.net/2433/218391

RIGHT:

(C) 2016 American Physical Society. 
PHYSICAL REVIEW D 94, 014007 (2016)

\title{
Models of the LHC diphoton excesses valid up to the Planck scale
}

\author{
Yuta Hamada, ${ }^{*}$ Hikaru Kawai, ${ }^{\dagger}$ Kiyoharu Kawana, ${ }^{*}$ and Koji Tsumura ${ }^{\S}$ \\ Department of Physics, Kyoto University, Kyoto 606-8502, Japan
}

(Received 2 March 2016; published 8 July 2016)

\begin{abstract}
We discuss a possibility to explain the LHC diphoton excesses at $750 \mathrm{GeV}$ by the new scalar $X$ that couples to the gauge bosons through the loop of new massive particles with standard model charges. We assume that the new particles decay into the standard model particles at the tree level. We systematically examine the models that preserve the vacuum stability and the perturbativity up to the Planck scale. When we take scalars for the new particles, we find that only a few diquark and dilepton models can explain the observed diphoton cross section without conflicting the experimental mass bounds. When we take vectorlike fermions for the new particles, we find rather different situations depending on whether their couplings to $X$ are scalar or pseudoscalar type. In the former case, a few models are allowed if we introduce only one species of fermions. The more fermions we introduce, the more models are allowed. In the latter case, most of the models are allowed because of the large coupling between $X$ and photon. It is interesting that the allowed mass regions of the scalar particles might be reached by the next lepton colliders.
\end{abstract}

DOI: 10.1103/PhysRevD.94.014007

\section{INTRODUCTION}

The standard model (SM) for elementary particles has been completed by the Higgs boson discovery. The observed properties of the Higgs boson is totally consistent with that in the SM, and no direct evidence of the new physics beyond the SM has been found at the Large Hadron Collider (LHC) so far. On the other hand, we know several indirect signatures of the beyond the SM, e.g., the existence of dark matter, smallness of the neutrino mass and the baryon asymmetry of the universe. Furthermore, there are many open questions in the SM as a consistent theory such as the naturalness problem of the Higgs boson mass [1,2], that of the cosmological constant [3], the origin of the electroweak symmetry breaking [4], and so on. To solve these questions, various models have been proposed. Each of them has its own theoretical motivations and characteristic phenomenological predictions. In order to disentangle these models, some experimental inputs of new physics is quite important.

Recently, both the ATLAS and CMS collaborations have reported the excesses of the signal events in the diphoton invariant mass distribution at around $750 \mathrm{GeV}$ based on the first results of the LHC Run 2 data [5,6]. Although these signal significances are around $3 \sigma$ confidence level, the results might be an indication of new physics beyond the SM. It would be valuable to study the interpretation of the observed excesses by simple scenarios because such a scenario can be understood as a low energy effective theory of more fundamental theories. By taking the Laudau-Yang

\footnotetext{
*hamada@gauge.scphys.kyoto-u.ac.jp

hkawai@gauge.scphys.kyoto-u.ac.jp

kiyokawa@gauge.scphys.kyoto-u.ac.jp

§ko2@gauge.scphys.kyoto-u.ac.jp
}

theorem $[7,8]$ into the consideration, a possible simple scenario is that the new particle $X$ with $M_{X} \sim 750 \mathrm{GeV}$ is a spin-zero SM singlet real scalar (or pseudoscalar) boson which couples to new massive scalar bosons or fermions charged under the SM gauge group [9]. ${ }^{1}$

Before going into details of the models, let us summarize the general aspects of $X$. When $X$ is a scalar, its effective couplings to the SM gauge bosons are parametrized as

$$
\mathcal{L}_{\text {eff }} \ni-\frac{X}{4}\left(g_{X \gamma} F^{\mu \nu} F_{\mu \nu}+g_{X g} G^{\mu \nu} G_{\mu \nu}\right)
$$

where the dimensionful parameters $g_{X \gamma}$ and $g_{X g}$ are determined by evaluating loop diagrams when we fix the model. Similarly, when $X$ is a pseudoscalar, the effective Lagrangian is given by

$$
\mathcal{L}_{\text {eff }} \ni-\frac{X}{4}\left(g_{X \gamma} F^{\mu \nu} \widetilde{F}_{\mu \nu}+g_{X g} G^{\mu \nu} \widetilde{G}_{\mu \nu}\right) .
$$

Here, $F^{\mu \nu}$ and $G^{\mu \nu}$ are the field strengths of the $U(1)_{\mathrm{em}}$ and $S U(3)_{c}$ respectively. Using these dimension-five interactions, the diphoton signal at the LHC can be interpreted as the process $g g \rightarrow X \rightarrow \gamma \gamma$ in Fig. 1 . The diphoton cross section $\sigma_{\gamma \gamma}$ is easily estimated by the gluon-fusion production cross-section times $\gamma \gamma$ branching ratio of $X$ at $13 \mathrm{TeV}$. In this paper, we follow the result in [11]:

\footnotetext{
${ }^{1}$ There can be other possible interpretations of the diphoton excesses. An interesting interpretation is that the peak in the diphoton is not a resonance of the new particle $X$ but a cusp in the loop integral of the box diagram of new particle $Y$ with $M_{Y} \sim 375 \mathrm{GeV}[10]$.
} 


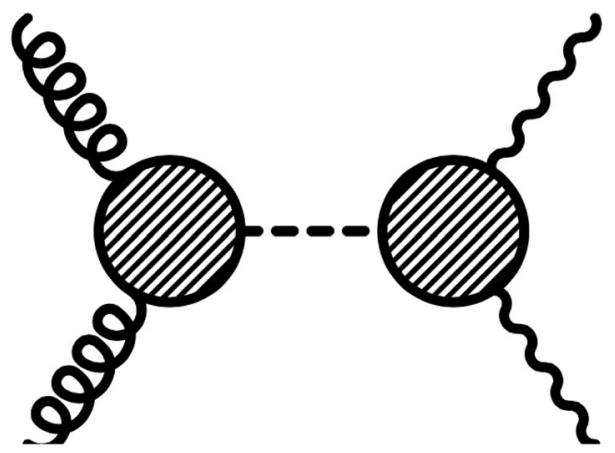

FIG. 1. A event topology of the simple interpretation for the LHC diphoton excess. The new particle $X$ with $M_{X}=750$ is produced by the gluon-fusion, and decays into a pair of photons.

$$
\begin{aligned}
\sigma_{\gamma \gamma} & :=\sigma(g g \rightarrow X) \times \operatorname{Br}(X \rightarrow \gamma \gamma) \\
& =(13 \mathrm{pb}) \times\left(g_{X \gamma} \cdot \mathrm{TeV}\right)^{2} .
\end{aligned}
$$

Note that the main decay mode of $X$ is assumed to be $g g$ channel. $^{2}$ Thus, the dependence of $g_{X g}$ in the overall reaction rate is almost canceled out. Throughout this paper, we assume the mass of internal particles in the loop to be heavier than the half of $M_{X}$ in order to forbid the tree level decay of $X$. On the other hand, the experimental data implies [11]

$$
\sigma_{\gamma \gamma}=1-10 \mathrm{fb} .
$$

By calculating the effective coupling $g_{X \gamma}$ in a specific model, we can compare Eq. (3) with Eq. (4) and discuss whether such a model is favorable in the context of the experimental data.

The coupling $g_{X \gamma}$ is generated at one-loop in each simple scenario. Thus, its magnitude is typically small due to not only the one-loop suppression but also the new mass scale. In order to generate sufficiently large $g_{X \gamma}$, we need relatively strong interactions among $X$ and the new SM charged particles. It is known that such strong interactions often violate theoretical consistencies of the model. For instance, a large trilinear scalar coupling $\mu$ tends to induce the vacuum instability. In order to rescue the vacuum stability, one may introduce a large scalar quartic coupling $\lambda_{\phi}$. However, such $\lambda_{\phi}$ leads to a rapid blowup of the running coupling constant and breaks the perturbativity below the Planck scale. Therefore, requirements of theoretical consistency give nontrivial constraints on the models of the diphoton excesses.

In this paper, we study whether simple scenarios can explain the diphoton excesses at the LHC while preserving the vacuum stability of the scalar potential and the perturbativity of the gauge, Yukawa and scalar quartic

\footnotetext{
${ }^{2}$ This assumption typically predicts the narrow decay width of $X$ which is favored by CMS data [6]. On the other hand, ATLAS results prefer the wide width of $\sim 45 \mathrm{GeV}$ [5].
}

coupling constants up to the Planck scale. ${ }^{3}$ As for the new SM charged particles, we consider scalars (diquark, leptoquark and dilepton) or fermions (vectorlike quark and vectorlike lepton) which can decay to the SM particles at tree level. For the completeness, we also consider the cases with $N_{f}$ multiplets of the new SM charged particles in order to maximize the diphoton cross sections. To study the perturbativity up to the Planck scale, we use the one-loop renormalization group equations (RGEs). If we consider a scalar $\left(N_{f}=1\right)$ as the new particles, only a few models can explain the observed cross section without conflicting the experimental mass bounds. The allowed models require diquarks and dileptons near the threshold masses $(\lesssim 450 \mathrm{GeV})$. All the leptoquark models are disfavored in our analysis due to the strong lower mass bounds at the LHC and the rapid running of the scalar quartic coupling constants. The increase of $N_{f}$ makes the situation better, however, too large $N_{f}$ is disfavored by the RGE of the quartic coupling of the new scalars. On the other hand, if we take vectorlike fermions as the new particles, the effective coupling $g_{X \gamma \gamma}$ behaves differently depending on the type of the Yukawa couplings between $X$ and the fermions. In the case of the scalar type coupling, only a few models are allowed if $N_{f}=1$. If we increase $N_{f}$, more models are allowed. Furthermore, in the case of the pseudoscalar type coupling, almost all of the models are allowed because of the enhancement of the effective coupling $g_{X \gamma \gamma}$ due to the property of the one-loop integral.

This paper is organized as follows. In Sec. II, we study the SM extended by $X$ and the new SM charged scalars. Stability and perturbativity bounds of the new scalar sector are discussed as well as the interpretation of the LHC diphoton excess. In Sec. III, the scenarios with new vectorlike fermions are considered in a similar manner. Conclusions and discussion are given in Sec. IV. In Appendix A, we summarize the direct search mass bounds for the new particles. In Appendix B, the one loop RGEs are presented.

\section{EXTENSIONS WITH NEW SCALAR BOSONS}

In this section, we study the extensions of the SM by new scalar multiplets with SM charges, which will be regarded as the source of $g_{X \gamma}$ via the one-loop diagram. Requiring tree-level decays of the new scalar bosons, ${ }^{4}$ their quantum charges of the new scalar fields are determined by the

${ }^{3}$ For the scenarios with the vectorlike fermions, the similar analysis was first done in [12] where they discuss the perturbativity and the vacuum stability of the models. Our analysis is more inclusive in that we also consider the extensions with the new SM charged scalars, and include the new scalar couplings. These couplings play important role to determine the Landau pole. See Sec. II for the details.

${ }^{4}$ The abundance of long-lived charged particles is strongly constrained by cosmology [13]. On the other hand, color singlet particles are allowed as a part of dark matter [14]. For those extensions, the following discussion can be equally applied. 
TABLE I. A list of diquarks which couple to the SM quarks. Here, $\left(N_{f}^{2} \lambda_{\phi}\right)^{\text {Max }}$ is the maximal value of $N_{f}^{2} \lambda_{\phi}^{\operatorname{Max}}$ where $\lambda_{\phi}^{\text {Max }}$ is calculated for each $N_{f}$ by requiring the perturbativity up to the Planck scale.

\begin{tabular}{lccrcr}
\hline \hline & $S U(3)_{c}$ & $S U(2)_{L}$ & $U(1)_{Y}$ & $\lambda_{\phi}^{\operatorname{Max}}$ & $\left(N_{f}^{2} \lambda_{\phi}\right)^{\operatorname{Max}}$ \\
\hline$D Q_{0}^{d}$ & $\mathbf{3}\left(\mathbf{6}^{*}\right)$ & $\mathbf{1}$ & $-1 / 3$ & $0.32(\times)$ & $\left.2.2\right|_{N_{f}=6}$ \\
$D Q_{0}^{y}$ & $\mathbf{3}\left(\mathbf{6}^{*}\right)$ & $\mathbf{1}$ & $-4 / 3$ & $0.34(\times)$ & $\left.1.2\right|_{N_{f}=3}$ \\
$D Q_{0}^{u}$ & $\mathbf{3}\left(\mathbf{6}^{*}\right)$ & $\mathbf{1}$ & $2 / 3$ & $0.33(\times)$ & $\left.2.2\right|_{N_{f}=6}$ \\
$D Q_{1}$ & $\mathbf{3}\left(\mathbf{6}^{*}\right)$ & $\mathbf{3}$ & $-1 / 3$ & $0.13(\times)$ & $\left.0.13\right|_{N_{f}=1}$ \\
$D Q_{1 / 2}$ & $\mathbf{1}_{\mathbf{H}}(\mathbf{8})$ & $\mathbf{2}$ & $1 / 2$ & $0.18(\times)$ & $\left.8.5\right|_{N_{f}=20}$ \\
\hline \hline
\end{tabular}

TABLE II. A list of leptoquarks which couple to the SM fermions.

\begin{tabular}{lcrrrr}
\hline \hline & $S U(3)_{c}$ & $S U(2)_{L}$ & $U(1)_{Y}$ & $\lambda_{\phi}^{\operatorname{Max}}$ & $\left(N_{f}^{2} \lambda_{\phi}\right)^{\operatorname{Max}}$ \\
\hline$S_{0}^{d *}$ & $\mathbf{3}^{*}$ & $\mathbf{1}$ & $1 / 3$ & 0.32 & $\left.2.2\right|_{N_{f}=6}$ \\
$S_{0}^{y *}$ & $\mathbf{3}^{*}$ & $\mathbf{1}$ & $4 / 3$ & 0.34 & $\left.1.2\right|_{N_{f}=3}$ \\
$S_{1}^{*}$ & $\mathbf{3}^{*}$ & $\mathbf{3}$ & $1 / 3$ & 0.13 & $\left.0.13\right|_{N_{f}=1}$ \\
$S_{1 / 2}$ & $\mathbf{3}$ & $\mathbf{2}$ & $7 / 6$ & 0.25 & $\left.0.46\right|_{N_{f}=2}$ \\
$S_{1 / 2}^{q}$ & $\mathbf{3}$ & $\mathbf{2}$ & $1 / 6$ & 0.24 & $\left.0.59\right|_{N_{f}=3}$ \\
$R_{0}^{u *}$ & $\mathbf{3}^{*}$ & $\mathbf{1}$ & $-2 / 3$ & 0.33 & $\left.2.2\right|_{N_{f}=6}$ \\
$R_{0}^{d *}$ & $\mathbf{3}^{*}$ & $\mathbf{1}$ & $1 / 3$ & 0.32 & $\left.2.2\right|_{N_{f}=6}$ \\
$R_{1 / 2}^{*}$ & $\mathbf{3}$ & $\mathbf{2}$ & $-1 / 6$ & 0.24 & $\left.0.59\right|_{N_{f}=3}$ \\
\hline \hline
\end{tabular}

combination of the SM fermions. We list all the possible charge assignments of the new scalar multiplets in Table I for diquarks, Table II for leptoquarks, and Table III for dileptons. Note that we include right-handed neutrinos $\nu_{R}$ as SM fermions. In these Tables, the normalization of the hypercharge is given by $I_{3}+Y_{\phi}=Q$.

The diquark field in Table I is $\mathbf{3}$ or $\mathbf{6}^{*}$ representation under the QCD as $\left(q \overline{q^{c}}\right)$ states and $\mathbf{1}$ or $\mathbf{8}$ representation as $(q \bar{q})$ states. The 1 representation is nothing but a Higgs doublet in the SM. Except for color charge assignments, there are five variations of diquarks. Each of them has specific decay pattern depending on the Yukawa couplings (see Appendix A). Eight possible leptoquark fields are listed in Table II. All of them are 3 representation under the color gauge group since they are $\left(q \overline{\ell^{c}}\right)$ or $(q \bar{\ell})$ states. In addition to the conventional scalar leptoquarks listed by PDG [15], we here include $R_{0}^{u}, R_{0}^{d}$, and

TABLE III. A list of dileptons which couple to leptons.

\begin{tabular}{lccccc}
\hline \hline & $S U(3)_{c}$ & $S U(2)_{L}$ & $U(1)_{Y}$ & $\lambda_{\phi}^{\operatorname{Max}}$ & $\left(N_{f}^{2} \lambda_{\phi}\right)^{\operatorname{Max}}$ \\
\hline$h_{0}^{+}$ & $\mathbf{1}$ & $\mathbf{1}$ & 1 & 0.25 & $\left.19\right|_{N_{f}=25}$ \\
$h_{0}^{++}$ & $\mathbf{1}$ & $\mathbf{1}$ & 2 & 0.27 & $\left.1.1\right|_{N_{f}=3}$ \\
$\Delta_{1}$ & $\mathbf{1}$ & $\mathbf{3}$ & 1 & 0.27 & $\left.0.77\right|_{N_{f}=3}$ \\
$\Phi_{1 / 2}$ & $\mathbf{1}_{\mathbf{H}}$ & $\mathbf{2}$ & $1 / 2$ & 0.25 & $\left.8.5\right|_{N_{f}=20}$ \\
$s_{0}$ & $\mathbf{1}$ & $\mathbf{1}$ & 0 & $\times$ & $\times$ \\
\hline \hline
\end{tabular}

$R_{1 / 2}$ leptoquarks since we regard $\nu_{R}$ as the SM fermions. All possible dilepton fields are given in Table III. In this class of extended models, we need to introduce additional colored particles $S_{c}$ in order to guarantee the sufficiently large production cross section of $g g \rightarrow X .^{5}$ In the following, we neglect the contribution to $g_{X \gamma}$ from $S_{c}$ for simplicity. This situation is realized if $S_{c}$ has a relatively small hypercharge. In Appendix A, we give a list of conservative estimates for the lower mass bounds of the new scalar bosons, which will be compared with the bound derived from theoretical considerations.

The scalar potential of each model is generally given by

$$
\begin{aligned}
V= & -\frac{M_{h}^{2}}{2}\left(H^{\dagger} H\right)+M_{\phi}^{2}\left(\phi^{\dagger} \phi\right)+\frac{M_{X}^{2}}{2} X^{2}+\mu\left(\phi^{\dagger} \phi\right) X \\
& +\mu^{\prime}\left(H^{\dagger} H\right) X+\frac{\mu_{X}}{3 !} X^{3}+\lambda\left(H^{\dagger} H\right)^{2}+\lambda_{\phi}\left(\phi^{\dagger} \phi\right)^{2} \\
& +\frac{\lambda_{X}}{4 !} X^{4}+\lambda_{\phi}^{\prime}\left(\phi^{\dagger} T_{\phi}^{A} \phi\right)^{2}+\lambda_{\phi}^{\prime \prime}\left(\phi^{\dagger} t_{\phi}^{a} \phi\right)^{2}+\lambda_{\phi}^{\prime \prime \prime}\left(\phi^{\dagger} T_{\phi}^{A} t_{\phi}^{a} \phi\right)^{2} \\
& +\lambda_{\mathrm{tr}}\left(\phi^{\dagger i} \phi_{j}\right)\left(\phi^{\dagger j} \phi_{i}\right)+\lambda_{\phi 2}\left(\phi^{\dagger}\left\{t_{\phi}^{a}, t_{\phi}^{b}\right\} \phi\right)^{2}+\lambda_{\mathrm{ad}} \operatorname{Tr}\left(\tilde{\phi}_{\alpha} \tilde{\phi}_{\beta}\right) \\
& \times \operatorname{Tr}\left(\tilde{\phi}^{\alpha^{\dagger}} \tilde{\phi}^{\beta \dagger}\right)+\lambda_{\mathrm{ad}}^{\prime} \operatorname{Tr}\left(\tilde{\phi}_{i}^{\prime} \tilde{\phi}_{j}^{\prime}\right) \operatorname{Tr}\left(\tilde{\phi}^{\prime \dagger \dagger} \tilde{\phi}^{\prime \dagger \dagger}\right) \\
& +\kappa_{H \phi}\left(H^{\dagger} H\right)\left(\phi^{\dagger} \phi\right)+\kappa_{H \phi}^{\prime}\left(H^{\dagger} t_{H}^{a} H\right)\left(\phi^{\dagger} t_{\phi}^{a} \phi\right) \\
& +\frac{\kappa_{H X}}{2}\left(H^{\dagger} H\right) X^{2}+\frac{\kappa_{\phi X}}{2}\left(\phi^{\dagger} \phi\right) X^{2}+\ldots,
\end{aligned}
$$

where $\phi$ is one of the scalar fields listed in Tables I-III, $i(j)$ is the $S U(2)_{L}$ index of $\phi, H$ is the SM Higgs doublet field, $t_{S}^{a}(a=1-3)$ is the $S U(2)_{L}$ generator for $S=H, \phi, T_{\phi}^{A}$ $(A=1-8)$ is the $S U(3)_{c}$ generator of $\phi$, and $\tilde{\phi}\left(\tilde{\phi}^{\prime}\right):=$ $\sum_{a} \phi^{a} t_{\phi}^{a}\left(\sum_{A} \phi^{A} T^{A}\right)$ exists only when the representation of $\phi$ is the adjoint representation. In the following discussion, we put $\mu^{\prime}=\mu_{X}=\lambda_{\phi}^{\prime}=\lambda_{\phi}^{\prime \prime}=\lambda_{\phi}^{\prime \prime \prime}=\lambda_{\text {tr }}=\lambda_{\phi 2}=\lambda_{\text {ad }}=\lambda_{\text {ad }}^{\prime}=$ $\kappa_{H \phi}=\kappa_{H \phi}^{\prime}=\kappa_{\phi X}=0$ at the weak scale for simplicity. ${ }^{6}$

\footnotetext{
${ }^{5}$ In principle, photon-fusion can be a source of $X$ production if we take photon into account as a parton [16]. However, it is required to have extremely large $g_{X \gamma}$ coupling at one-loop level.

${ }^{6}$ These couplings and a large number of flavors (particles) can potentially bring some conflicts with the electroweak precision test (EWPT) [17]. For example, the mixing coupling $\kappa_{H \phi}^{\prime}$ generates the mass splitting of $\phi$, and contributes to the oblique parameters. Thanks to our simplified choice of parameters, the constraints from the EWPT are evaded. Furthermore, as for the cubic couplings, they can induce a nonzero vacuum expectation value (vev) of $X$. Such a vev can generally decrease $\sigma_{\gamma \gamma}$ because the total decay width of $X$ becomes larger through the possible direct decay $X \rightarrow \bar{H} H$. As for the scalar quartic couplings, there might be parameter regions such that $\sigma_{\gamma \gamma}$ becomes larger. For example, when the mixing couplings such as $\kappa_{H \phi}$ are negative, the vacuum stability is not guaranteed any more, but we can rescue it by making other quartic self-couplings positively large. In principle, such choices can increase the upper bound of $\mu$ [see Eq. (9)]. However, we must simultaneously consider the RGE effects of these couplings, and such effects can be stronger than the former. Seeking for some fine-tuned parameter regions is beyond the one-loop analysis of this paper.
} 
As we noted in the Introduction, the diphoton decay of $X$ is induced at the one-loop level. The effective coupling $g_{X \gamma}$ is calculated as

$$
g_{X \gamma}=\frac{N_{f} \alpha \sum_{\phi} Q_{\phi}^{2}}{2 \pi} \frac{\mu}{M_{X}^{2}} f\left(M_{\phi}^{2} / M_{X}^{2}\right)
$$

where $\alpha\left(=e^{2} / 4 \pi\right)$ is the fine-structure constant, $\sum_{\phi} Q_{\phi}^{2}$ stands for summation of $U(1)_{\mathrm{em}}$ charge squared over the multiplet $\phi$ including the color factor, and $N_{f}$ denotes the number of flavors of $\phi$. Because we simplify the scalar potential by taking $\kappa_{H \phi}=\kappa_{H \phi}^{\prime}=0$, components of $\phi$ are degenerate in mass $M_{\phi}$. The one-loop integral is easily evaluated as

$$
f(x):=8 x\left(\arctan \frac{1}{\sqrt{4 x-1}}\right)^{2}-2 .
$$

The function takes the maximal value $f(1 / 4)=\left(\pi^{2}-4\right) / 2$ at the threshold $M_{X}=2 M_{\phi}$, and $\phi$ decouples monotonically for large $M_{\phi}$, i.e., $f(\infty)=0$.

In order to obtain the large diphoton cross section, the scalar trilinear coupling $\mu$ is required to be large as in Eq. (6). On the other hand, too large $\mu$ is constrained by the vacuum stability. By focusing on $M_{X}^{2}, \mu$ and $\lambda_{\phi}$ terms in Eq. (5), we have

$$
\begin{aligned}
V & \ni \frac{M_{X}}{2} X^{2}+\mu\left(\phi^{\dagger} \phi\right) X+\lambda_{\phi}\left(\phi^{\dagger} \phi\right)^{2} \\
& =\frac{M_{X}^{2}}{2}\left(X+\frac{\mu}{M_{X}^{2}}\left(\phi^{\dagger} \phi\right)\right)^{2}+\left(\lambda_{\phi}-\frac{\mu^{2}}{2 M_{X}^{2}}\right)\left(\phi^{\dagger} \phi\right)^{2},
\end{aligned}
$$

which leads to

$$
\mu^{2}<2 \lambda_{\phi} M_{X}^{2}
$$

by imposing the stability of the scalar potential at tree level. ${ }^{7}$ Equation (9) shows that large $\lambda_{\phi}$ can help the vacuum stability against $\mu$.

Furthermore, the magnitude of $\lambda_{\phi}$ is also constrained by requiring the perturbativity up to the Planck scale. It is known that a large quartic coupling constant is a source of the Landau pole at high energy by considering the renormalization group evolution. In our setup, in order to ensure the substantial diphoton signal rate, small $\lambda_{\phi}$ is disfavored through the vacuum stability condition. Therefore, $\lambda_{\phi}$ tends to be nonperturbative at high energy. In Tables I, II and III, we list $\lambda_{\phi}^{\mathrm{Max}}$ (the maximal values of $\lambda_{\phi}$

\footnotetext{
${ }^{7}$ If $X$ has a nonzero vacuum expectation value $\langle X\rangle$, the $\left(\phi^{\dagger} \phi\right) X$ coupling gets another contribution from $\left(\phi^{\dagger} \phi\right) X^{2}$. However, the total effective coupling $\tilde{\mu}=\mu+\langle X\rangle \kappa_{\phi X} / 2$ also follows the same equation as Eq. (9). Therefore, our examination Eq. (10) does not change. Moreover, nonzero $\langle X\rangle$ opens up new decay channels such as $X \rightarrow H H$ through the $\left(H^{\dagger} H\right) X^{2}$ coupling, which leads to the decrease of $\sigma_{\gamma \gamma}$. Thus, to maximize $\sigma_{\gamma \gamma}$, we assume $\langle X\rangle=0$ in this paper.
}

at weak scale), which keeps the purturbativity up to the Planck scale, in various scalar extended models. The values are calculated using the one-loop RGEs in Appendix B. ${ }^{8}$ On one hand, to maximize the diphoton signal rate, larger $N_{f}$ seems to be favorable. On the other hand, the perturbativity bounds become more severe. We determine $\left(N_{f}^{2} \lambda_{\phi}\right)^{\operatorname{Max}}$, the maximal value of $N_{f}^{2} \lambda_{\phi}^{\max }$ where $\lambda_{\phi}^{\operatorname{Max}}$ is calculated for each $N_{f}$ by requiring the pertubativity condition up to the Planck scale. In the following, we denote the corresponding $N_{f}$ as $N_{\mathrm{Max}}$. Notice that when the $S U(3)_{c}$ representation of $6^{*}$ and 8 , the Landau pole of $\lambda_{\phi}$ appears below the Planck scale even if $\lambda_{\phi}$ is set to be zero at the weak scale, see also Ref. [18] for the $S U(2)$ case.

Combining Eqs. (3), (6), and (9), we calculate the maximum value of $\sigma_{\gamma \gamma}$ :

$$
\begin{aligned}
\sigma_{\gamma \gamma}^{\operatorname{Max}}= & 46 \mathrm{pb} \times \lambda_{\phi}^{\operatorname{Max}} \times\left(\frac{N_{f} \alpha \sum_{\phi} Q_{\phi}^{2}}{2 \pi} f\left(M_{\phi}^{2} / M_{X}^{2}\right)\right)^{2} \\
& \times\left(\frac{750 \mathrm{GeV}}{M_{X}}\right)^{2} .
\end{aligned}
$$

In Fig. 2, we plot the maximal $\sigma_{\gamma \gamma}$ in Eq. (10) as functions of $M_{\phi}$ for various scalar extended models. The left (right) panels show the case with $N_{f}=1\left(N_{\text {Max }}\right)$. The labels $\left(R_{c}, 2 I+1, Y_{\phi}\right)$ denote the representation of the new scalar under the $S U(3)_{c}, S U(2)_{L}$, and $U(1)_{Y}$. The dashed part of the curves indicates the excluded mass range from the direct collider searches for $N_{f}=1$ (see Appendix A). For $N_{f}=N_{\max }$, these maximal cross sections are given by the dotted curves, since we do not know the corresponding experimental bounds (although some of them can be estimated). The shaded region is $\sigma_{\gamma y}^{\mathrm{Max}}>1 \mathrm{fb}$, which can be consistent with the LHC diphoton excesses.

In the top panels, the maximal value of $\sigma_{\gamma \gamma}$ is plotted for each diquark model. The models with $\mathbf{6}^{*}$ and $\mathbf{8}$ representations under the QCD are not presented because these models do not keep the perturbativity up to the Planck scale. For diquarks of the 3 representations, only two representations of $(3,1,-4 / 3)$ and $(3,3,-1 / 3)$ with $N_{f}=1$ can be consistent with the perturbativity and the LHC diphoton excess. If $N_{f}>1$, the maximal cross sections are getting larger because these are proportional to $N_{f}^{2}$. On the other hand, excessively large $N_{f}$ leads the breakdown of the perturbativity of $\lambda_{\phi}$ before the Planck scale. Even when the perturbativety is kept, the allowed maximal value of the scalar trilinear coupling $\mu$ becomes smaller. Furthermore, the increase of $N_{f}$ makes the

\footnotetext{
${ }^{8}$ In our calculation, the contributions from the possible Yukawa couplings are neglected. Indeed, the new particles decay promptly even for very small Yukawa couplings. On the other hand, if the Yukawa couplings are large, $\lambda_{\phi}^{\mathrm{Max}}$ would be increased.
} 
MODELS OF THE LHC DIPHOTON EXCESSES VALID UP ...
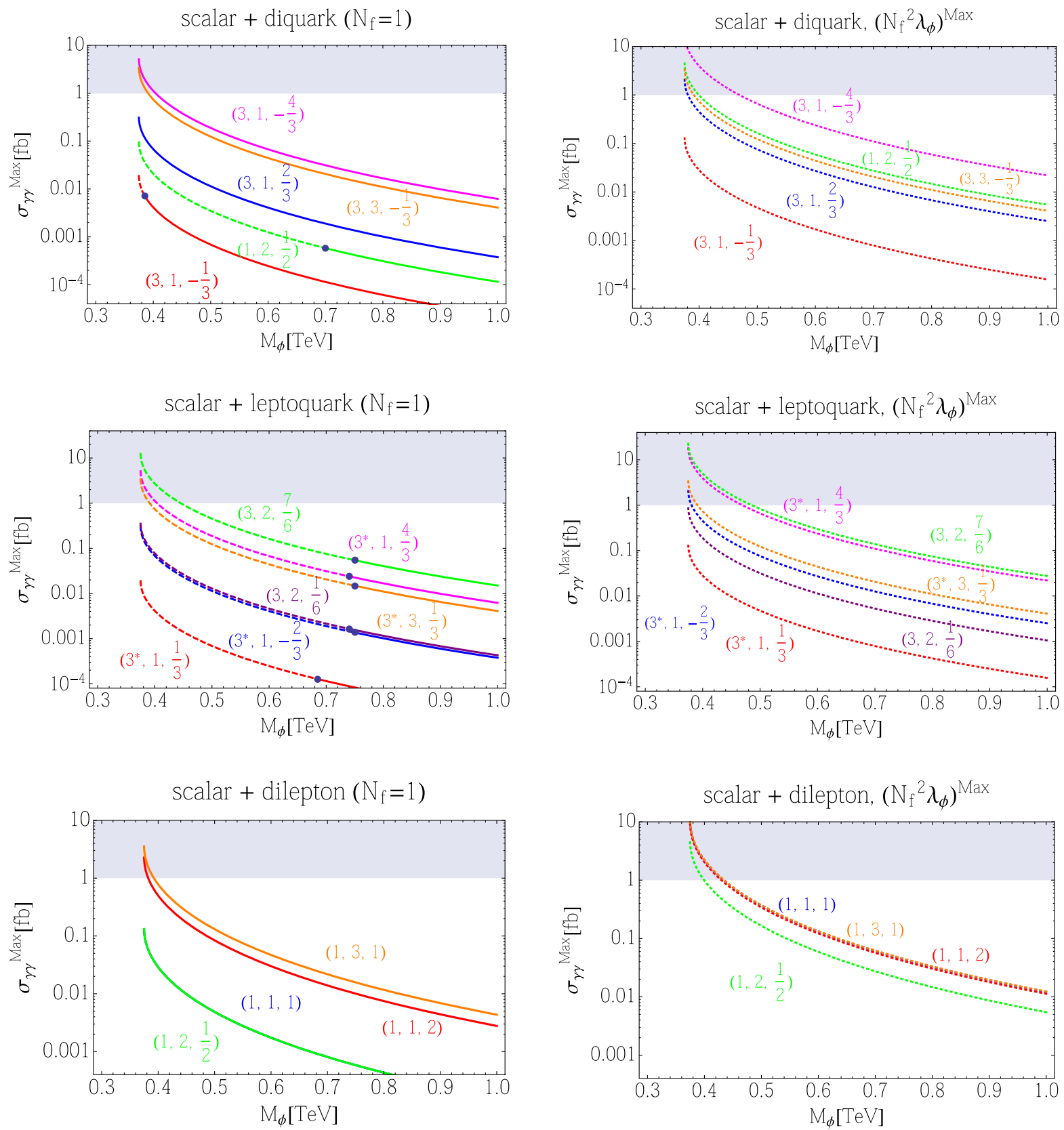

FIG. 2. The maximal value, $\sigma_{\gamma \gamma}^{\mathrm{Max}}$ for the production cross sections times $\gamma \gamma$ branching ratios, are shown as functions of $M_{\phi}$, the mass of the new SM charged scalar boson. Each curve shows the value calculated in the different scalar extension models. The solid (dashed) part of curves is the allowed (excluded) mass range by the collider experiments for $N_{f}=1$. For $N_{f}>1$, the results are given by dotted curves. The shaded region implies the required cross section from the LHC diphoton excess, i.e., $\sigma_{\gamma \gamma}^{\mathrm{Max}}>1 \mathrm{fb}$.

experimental bound stronger, so that the situation does not always become better.

For the leptoquark extensions (middle panels), some of the models can predict large enough $\sigma_{\gamma \gamma}$, however, all the representations suffer from severe direct search constraints. For $N_{f}=1$, no model fulfills the simultaneous requirements of the perturbativity and the LHC diphoton excess assuming that the leptoquarks only couple to one generation of the SM fermions. The situation is not improved for $N_{f}>1$, because of the stringent experimental bounds.
A leptoquark which mainly decays to a light-quark and a tau lepton pair is less constrained because of the difficulty of their identifications. To our knowledge, no corresponding constraint on such a leptoquark is found, and it might be consistent with these conditions.

For the dilepton extended models, the values of $\sigma_{\gamma \gamma}$ are relatively small as compared with the colored scalar models due to the small degrees of freedom. However, thanks to the weaker experimental mass bounds, two representations of $(1,3,1)$ and $(1,1,2)$ including the doubly charged Higgs 
boson survive both the experimental and theoretical constraints for $N_{f}=1$. For larger $N_{f}$, all models can predict a sufficiently large diphoton cross section with lighter dileptons. Because of the clean signal of leptonic observable, large $N_{f}$ would be easily excluded. It would be nice if the experimental groups release the results for larger mass regions.

From these plots, we find that only a few models with $N_{f}=1$ can explain the LHC diphoton excess without conflicting the requirement of the perturbativity up to the Planck scale. These conditions typically favor the new scalar field with a higher representation of $S U(2)_{L}$ and a large hypercharge. In all the allowed models, the required scalars are relatively light, $M_{\phi} \lesssim 450 \mathrm{GeV}$. Its precision measurement would be a good target at the ILC with the CM energy of $1 \mathrm{TeV}$. The doubly charged scalars in the dilepton multiplets may also be produced at the $e^{-} e^{-}$option of the ILC if the relevant Yukawa coupling is sufficiently large.

\section{EXTENSIONS WITH NEW VECTORLIKE FERMIONS}

In this section, we consider vectorlike fermions instead of the scalar multiplets discussed in the previous section. The new fermions are used to be a source of $g_{X \gamma}$ at one-loop level. Similarly to the scalars, the representations of the vectorlike fermions under the SM gauge group are fully determined by allowing the tree level decays to the SM particles. Their quantum charges are listed in Table IV for vectorlike quarks and in Table $\mathrm{V}$ for vectorlike leptons.

Because all vectorlike fermions listed here decay to the Higgs field and the SM fermions, they must be color triplet or singlet. Therefore, the allowed decay modes are SM fermions plus $W, Z$ or $H_{125}$. Small Yukawa couplings to the SM fields are sufficient for the prompt decay of the new fermions, so that we neglect them in the following discussion, i.e., masses of each component of vectorlike fermions are degenerate. ${ }^{9}$ The experimental bounds on these vectorlike fermion masses are summarized in Appendix A. For the leptonic extended models, we implicitly assume the existence of a new colored particle (with relatively small hypercharge) similarly to the color-singlet scalar extensions.

Let us move to the calculation of the diphoton signals of $X$. In the following, we denote one of the vectorlike fermions in Tables IV and V by $\psi$. We consider the following two typical Yukawa interactions between $X$ and $\psi$ :

$$
\mathcal{L} \ni-M_{\psi} \bar{\psi} \psi-\left\{\begin{array}{ll}
y_{\psi} X \bar{\psi} \psi & (\text { scalar type }) \\
i y_{\psi} X \bar{\psi} \gamma_{5} \psi & (\text { pseudoscalar type })
\end{array} .\right.
$$

Because of this new Yukawa coupling, $X$ can decay to $\gamma \gamma$ at the one-loop level. The effective coupling $g_{X \gamma}$ is as follows:

\footnotetext{
${ }^{9}$ As a result, the contributions to the electroweak precision test are also negligible.
}

TABLE IV. A list of vector-like quarks which couple to the SM fields. For $T_{1 / 2}$, the Landau pole of $g_{Y}$ appears below the Planck scale even for $N_{f}=1$ [19].

\begin{tabular}{lcrrlc}
\hline \hline VLQs & $S U(3)_{c}$ & $S U(2)_{L}$ & $U(1)_{Y}$ & $y_{\psi}^{\operatorname{Max}}$ & $\left.\left(y_{\psi}^{\operatorname{Max}} N_{f}\right)\right|_{N_{f}=N_{\text {Max }}}$ \\
\hline$T_{0}$ & $\mathbf{3}$ & $\mathbf{1}$ & $2 / 3$ & 0.84 & $\left.2.5\right|_{N_{\text {Max }}=5}$ \\
$B_{0}$ & $\mathbf{3}$ & $\mathbf{1}$ & $-1 / 3$ & 0.82 & $\left.5.1\right|_{N_{\text {Max }}=13}$ \\
$T_{1}$ & $\mathbf{3}$ & $\mathbf{3}$ & $2 / 3$ & 0.80 & $\left.0.80\right|_{N_{\text {Max }}=1}$ \\
$B_{1}$ & $\mathbf{3}$ & $\mathbf{3}$ & $-1 / 3$ & 0.79 & $\left.0.79\right|_{N_{\text {Max }}=1}$ \\
$Q_{1 / 2}$ & $\mathbf{3}$ & $\mathbf{2}$ & $1 / 6$ & 0.72 & $\left.1.8\right|_{N_{\text {Max }}=4}$ \\
$T_{1 / 2}$ & $\mathbf{3}$ & $\mathbf{2}$ & $7 / 6$ & LP of $g_{Y}$ & LP of $g_{Y}$ \\
$B_{1 / 2}$ & $\mathbf{3}$ & $\mathbf{2}$ & $-5 / 6$ & 0.76 & $\left.0.76\right|_{N_{\text {Max }}=1}$ \\
\hline \hline
\end{tabular}

TABLE V. A list of vectorlike leptons which couple to the SM fields. For $N_{0}, y_{\psi}^{\mathrm{Max}}$ is not presented because it is a SM singlet.

\begin{tabular}{lccccc}
\hline \hline VLLs & $S U(3)_{c}$ & $S U(2)_{L}$ & $U(1)_{Y}$ & $y_{\psi}^{\operatorname{Max}}$ & $\left.\left(y_{\psi}^{\operatorname{Max}} N_{f}\right)\right|_{N_{f}=N_{\text {Max }}}$ \\
\hline$N_{0}$ & $\mathbf{1}$ & $\mathbf{1}$ & 0 & $\times$ & $\times$ \\
$E_{0}$ & $\mathbf{1}$ & $\mathbf{1}$ & -1 & 0.73 & $\left.2.9\right|_{N_{\text {Max }}=7}$ \\
$N_{1}$ & $\mathbf{1}$ & $\mathbf{3}$ & 0 & 0.77 & $\left.1.8\right|_{N_{\text {Max }}=3}$ \\
$E_{1}$ & $\mathbf{1}$ & $\mathbf{3}$ & -1 & 0.83 & $\left.1.4\right|_{N_{\text {Max }}=2}$ \\
$L_{1 / 2}$ & $\mathbf{1}$ & $\mathbf{2}$ & $-1 / 2$ & 0.68 & $\left.3.6\right|_{N_{\text {Max }}=12}$ \\
$E_{1 / 2}$ & $\mathbf{1}$ & $\mathbf{2}$ & $-3 / 2$ & 0.82 & $\left.0.82\right|_{N_{f}=1}$ \\
\hline \hline
\end{tabular}

$$
\begin{aligned}
g_{X \gamma}= & \frac{2 N_{f} \alpha \sum_{\psi_{i}} Q_{\psi_{i}}^{2} \frac{y_{\psi}}{M_{X}}}{\pi} \\
& \times \begin{cases}f_{S}\left(M_{\psi}^{2} / M_{X}^{2}\right) & (\text { scalar type }) \\
f_{P S}\left(M_{\psi}^{2} / M_{X}^{2}\right) & \text { (pseudoscalar type) }\end{cases}
\end{aligned}
$$

where

$f_{S}(x):=2 \sqrt{x}\left((1-4 x) \arctan \left(\frac{1}{\sqrt{4 x-1}}\right)^{2}+1\right)$,

and

$$
f_{P S}(x):=2 \sqrt{x} \arctan \left(\frac{1}{\sqrt{4 x-1}}\right)^{2} .
$$

Both functions take the maximal value at the threshold, $f_{S}(1 / 4)=1$ and $f_{P S}(1 / 4)=\pi^{2} / 4$, and decrease as $f_{S} \sim 1 /(3 \sqrt{x})$ and $f_{P S} \sim 1 /(2 \sqrt{x})$ for large $x$. As a result, for the pseudoscalar case, $\sigma_{\gamma \gamma}$ is about $\left(\pi^{2} / 4\right)^{2} \approx 6$ times larger than that of the scalar case around the threshold, and $(3 / 2)^{2}=2.25$ times larger in the heavy fermion limit.

As well as the scalar extension cases, the maximal value of $y_{\psi}$ can be determined by imposing the perturbativity up to the Planck scale. The one-loop RGE of $y_{\psi}$ is given by

$$
\frac{d y_{\psi}}{d t}=\frac{y_{\psi}}{16 \pi^{2}}\left(\left(6 n_{\psi} N_{f}+3\right) y_{\psi}^{2}-6 g_{2}^{2} C_{2}\left(n_{\psi}\right)-6 g_{Y}^{2} Y_{\psi}^{2}-8 g_{3}^{2}\right)
$$


MODELS OF THE LHC DIPHOTON EXCESSES VALID UP ...

PHYSICAL REVIEW D 94, 014007 (2016)
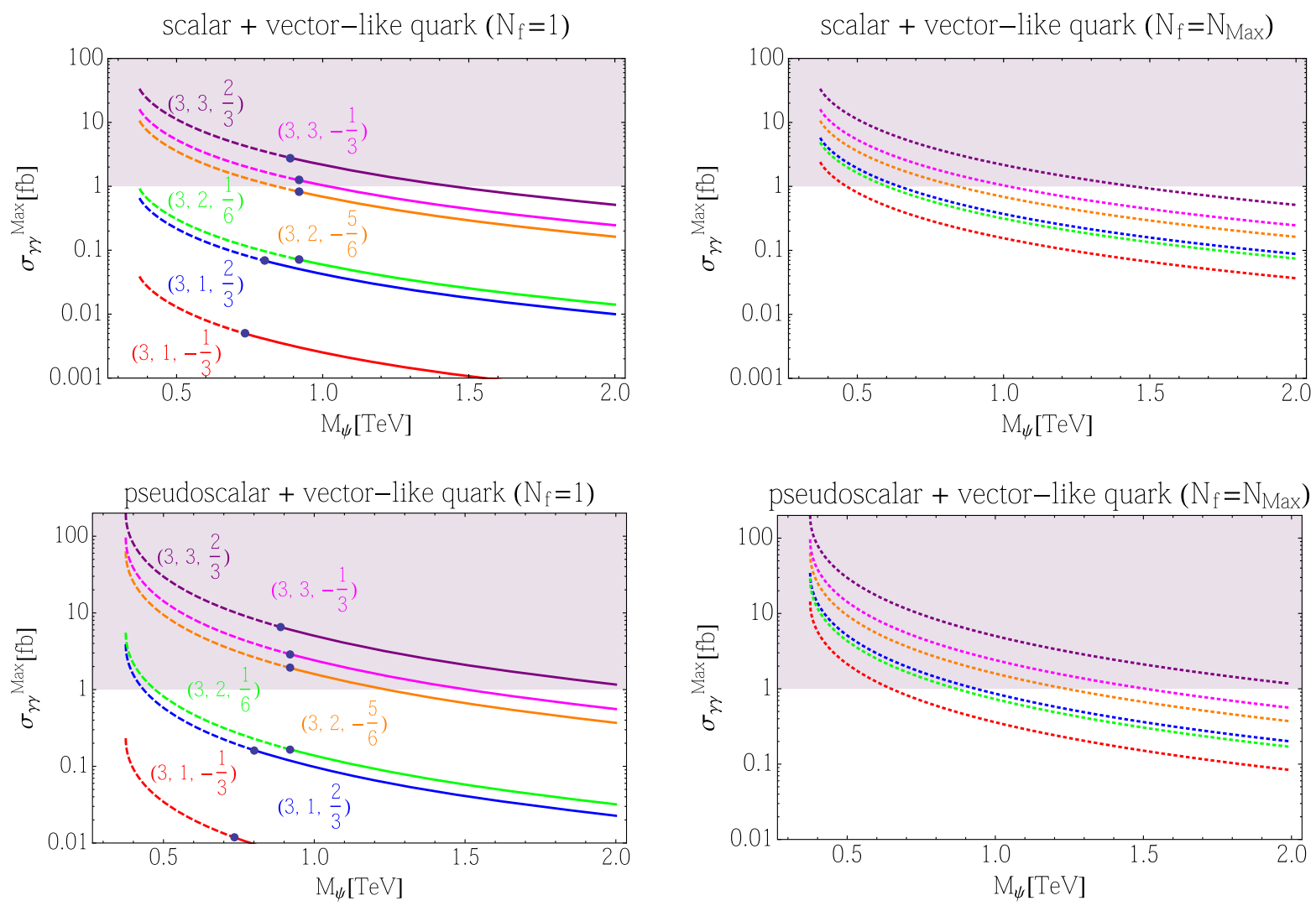

FIG. 3. The maximal cross sections $\sigma_{\gamma \gamma}^{\mathrm{Max}}$ in vectorlike quark extension models are given as functions of the mass $M_{\psi}$ of the vectorlike quarks. The curves and shaded region are shown in a similar manner to the scalar extensions.

for vectorlike quarks, and

$\frac{d y_{\psi}}{d t}=\frac{y_{\psi}}{16 \pi^{2}}\left(\left(2 n_{\psi} N_{f}+3\right) y_{\psi}^{2}-6 g_{2}^{2} C_{2}\left(n_{\psi}\right)-6 g_{Y}^{2} Y_{\psi}^{2}\right)$

for vectorlike leptons. Here, $n_{\psi}\left(=2 I_{\psi}+1\right)$ is the $S U(2)_{L}$ representation of $\psi, C_{2}$ is the Casimir index, and $Y_{\psi}$ is the $U(1)_{Y}$ hypercharge of $\psi$. The RGEs of the gauge couplings are presented in Appendix B.

In Tables IV and V, we list $y_{\psi}^{\mathrm{Max}}$ (the maximal values of $y_{\psi}$ at the weak scale) in various vectorlike fermion models. For these models, $N_{\text {Max }}$ is determined by the perturbativity bound of the SM gauge couplings. ${ }^{10}$ We then evaluate $y_{\psi}^{\mathrm{Max}}$ for $N_{f}=N_{\mathrm{Max}}$. The corresponding maximal values of $\sigma_{\gamma \gamma}$ can be calculated by substituting $y_{\psi}^{\mathrm{Max}}$ in Eq. (12) and using Eq. (3).

In Figs. 3 and 4, we show the maximal cross sections $\sigma_{\gamma \gamma}^{\mathrm{Max}}$ in the vectorlike quark and lepton extension models as functions of $M_{\psi}$, respectively. For the left (right) panel, the results for $N_{f}=1\left(N_{\text {Max }}\right)$ are given similarly to the figures of the scalar extended models. The two cases of the scalar

\footnotetext{
${ }^{10}$ Here, note that $N_{\text {Max }}$ is determined independently of $y_{\psi}$ because the Landau pole of $y_{\psi}$ never appears as long as it is zero at the weak scale.
}

and pseudoscalar Yukawa couplings, are presented in the upper and lower panels.

In the vectorlike quark extensions, we do not show the result for the $(3,2,7 / 6)$ representation because the Landau pole appears in the running of the hypercharge gauge coupling. For the rest, if the Yukawa coupling is the scalar type, two representations of $(3,3,2 / 3)$ and $(3,3,-1 / 3)$ are consistent with the requirements of the perturbativity, the explanation of the LHC diphoton excess, and the LHC direct search bound. In the case of the pseudoscalar type, thanks to the enhancement in the loop integral, a representation of $(3,2,-5 / 6)$ is rescued even when $N_{f}=1$. Furthermore, two more representations of $(3,2,1 / 6)$ and $(3,1,2 / 3)$ are saved by considering many flavors. In contrast to the scalar extensions, the situation gets better for large $N_{f}$, because $y_{\psi}^{\mathrm{Max}}$ is not so sensitive to $N_{f} \cdot{ }^{11} \mathrm{It}$ should be noted that the decoupling behavior is slower than the case of the scalar extension due to the different loop

\footnotetext{
${ }^{11}$ The reason is as follows. When $y_{\psi}$ and $N_{f}$ are relatively large, the RGE of $y_{\psi}$ is roughly $d y_{\psi} / d t \sim N_{f} y_{\psi}^{3} / 16 \pi^{2}$ from Eq. (15) or Eq. (16). However, by the redefinition $y_{\psi}:=N_{f}^{-1 / 2} \tilde{y}_{\psi}$, we can see that $\tilde{y}_{\psi}$ follows the RGE when $N_{f}=1$. Therefore, $y_{\psi}^{\operatorname{Max}}$ is roughly given by $N_{f}^{-1 / 2} \tilde{y}_{\psi}=\left.N_{f}^{-1 / 2} y_{\psi}^{\operatorname{Max}}\right|_{N_{f}=1}$, and the effect of $N_{f}$ is not much strong.
} 
HAMADA, KAWAI, KAWANA, and TSUMURA
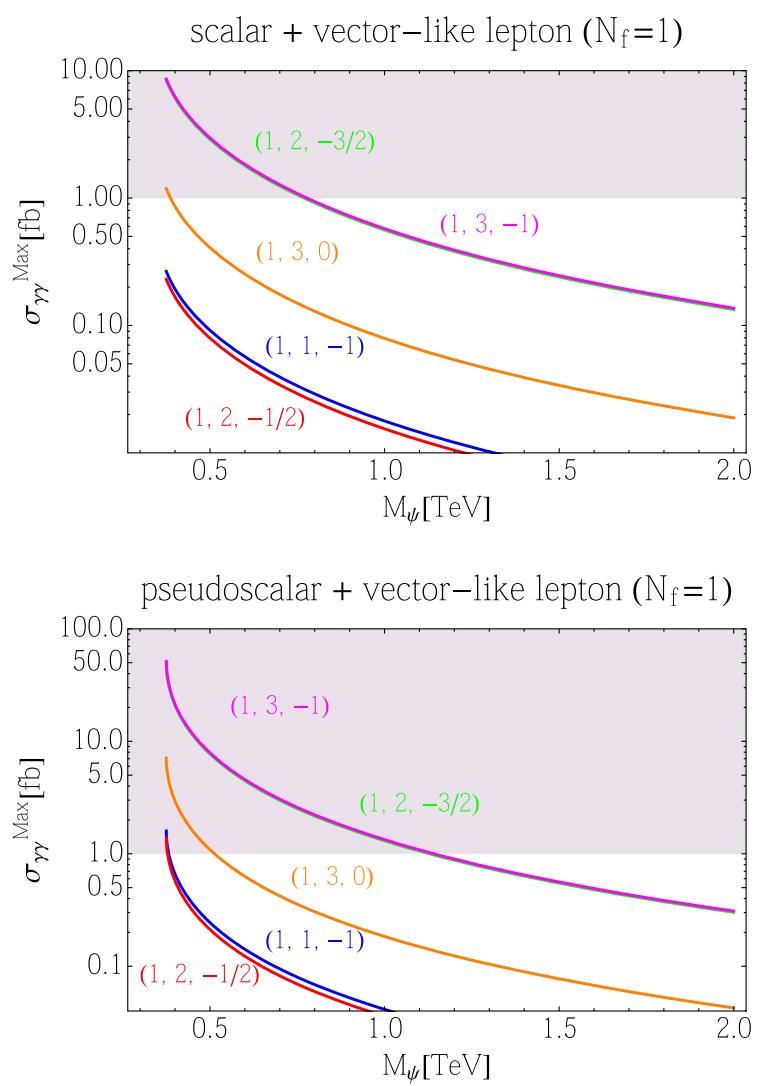

PHYSICAL REVIEW D 94, 014007 (2016)
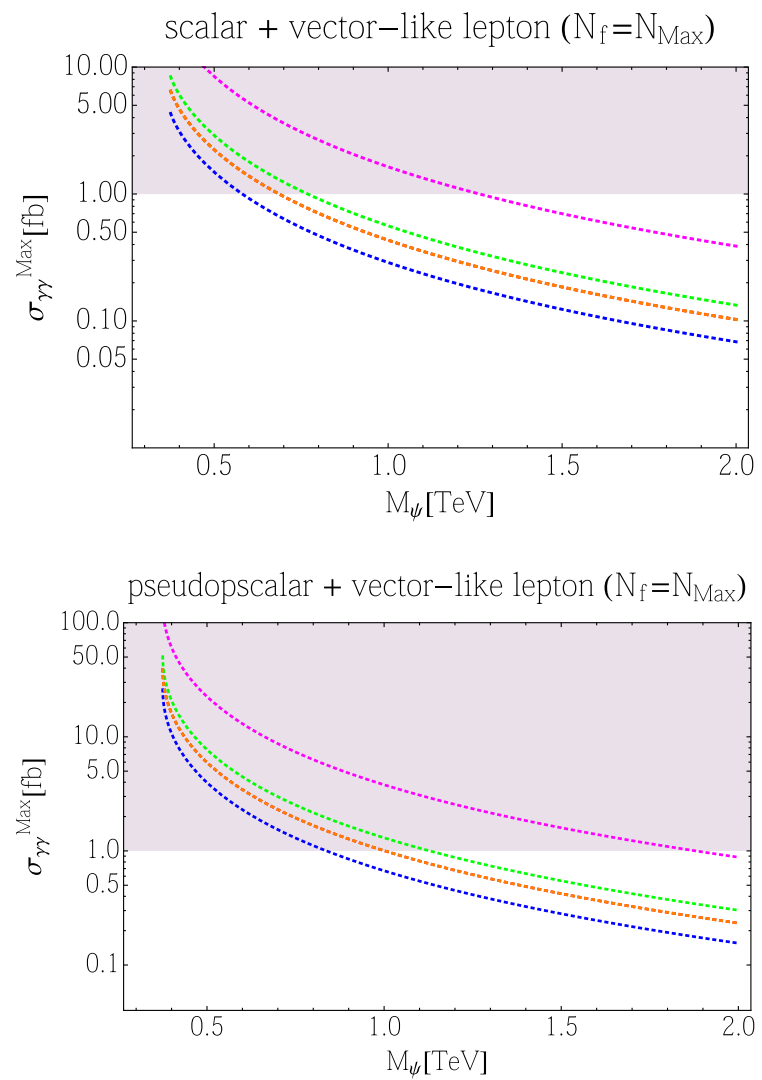

FIG. 4. The maximal cross sections $\sigma_{\gamma \gamma}^{\mathrm{Max}}$ in the vectorlike lepton extension models are presented as functions of the mass $M_{\psi}$ of the vectorlike leptons.

integral, so that relatively heavy vectorlike quark can predict sufficiently large $\sigma_{\gamma \gamma}$.

For vectorlike leptons, the experimental mass bounds are very loose because of the small production cross section. As we summarize in Appendix A, only the electron and muon decay channels have been searched. All of the vectorlike leptons could be as light as a half of the diphoton resonance mass $M_{X}$. As in the left-top panel of Fig. 4, the representations of $(1,2,-3 / 2),(1,3,-1)$, and $(1,3,0)$ can be consistent with all conditions for the scalar type Yukawa coupling with $N_{f}=1$. For the pseudoscalar case, all the models survive thanks to the largeness of $f_{P S}$, Eq. (14). By considering $N_{f}>1$, the required cross sections are easily satisfied.

These fermionic extensions only demand relatively heavy fermions unlike scalar extended models. Such heavy fermions would be beyond the scope at the LHC. In order to test the origin of the LHC diphoton excess completely, we may need to go to a further high energy frontier.

\section{CONCLUSIONS AND DISCUSSION}

We have studied perturbatively safe scenarios for the LHC diphoton excesses. The excess can be interpreted by a new scalar boson $X$ with $M_{X}=750 \mathrm{GeV}$ which decays to a pair of photons. A simple scenario is realized by introducing new scalar bosons or fermions charged under the
SM gauge group. The effective dimension-five interactions of $X$ and photons (gluons) are induced at one-loop of the new particles. In order to generate a sufficiently large effective interaction, large coupling constants are needed between $X$ and the new particles. On the other hand, such coupling constants are severely constrained by the theoretical consistencies of the model; We have investigated the stability bound of the scalar potential and the pertubativity conditions up to the Planck scale.

We have considered the cases with diquark, leptoquark, and dilepton multiplets for the scalar extensions, where all scalars can decay into the SM fermions at tree level. Some of the diquark and dilepton extensions can be consistent with all theoretical and experimental requirements while all the leptoquark extensions are ruled out due to the stringent direct search bounds. Increasing the number of new scalars helps the situation better if we choose appropriate $N_{f}$. All allowed models predict the new light scalar boson with a mass less than $450 \mathrm{GeV}$, which would be a very nice scope of the ILC.

We have also examined the vectorlike fermion extensions, which can mix the SM fermions through the mass matrix. For fermionic extensions, most models can simultaneously realize the LHC diphoton excesses and the perturbativity up to the Planck scale without contradicting the direct search mass bounds. A larger cross section for $\sigma_{\gamma \gamma}$ 
TABLE VI. Lower mass bounds for the diquark multiplets. The numbers in the parenthesis are our estimated bounds from the scaling of the cross sections for the color triplet diquark.

\begin{tabular}{|c|c|c|c|}
\hline & Decay modes & $M_{1 \mathrm{st}, 2 \mathrm{nd}} / \mathrm{GeV} \gtrsim$ & $M_{3 \mathrm{rd}} / \mathrm{GeV} \gtrsim$ \\
\hline \multirow[b]{2}{*}{$D Q_{0}^{d}$} & \multirow{2}{*}{ 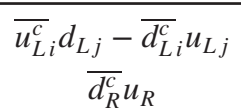 } & $350_{\mathbf{3}}(\sim 700)_{\mathbf{6}}[21]$ & N.A. \\
\hline & & $350_{\mathbf{3}}(\sim 700)_{\mathbf{6}}[21]$ & $385_{3}(\sim 700)_{6}[21]$ \\
\hline$D Q_{0}^{y}$ & $\overline{u_{R}^{c}} u_{R}$ & $350_{\mathbf{3}}(\sim 700)_{\mathbf{6}}[21]$ & $350_{\mathbf{3}}(\sim 700)_{6}[21]$ \\
\hline$D Q_{0}^{u}$ & $\overline{d_{R}^{c}} d_{R}$ & $350_{\mathbf{3}}(\sim 700)_{\mathbf{6}}[21]$ & $350_{\mathbf{3}}(\sim 700)_{6}[21]$ \\
\hline \multirow[t]{2}{*}{$D Q_{1}$} & 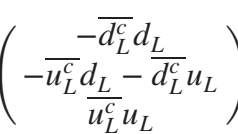 & $\left(\begin{array}{l}350_{\mathbf{3}}(\sim 700)_{\mathbf{6}}[21] \\
350_{\mathbf{3}}(\sim 700)_{\mathbf{6}}[21] \\
350_{\mathbf{3}}(\sim 70)_{\mathbf{6}}[21]\end{array}\right)$ & $\left(\begin{array}{l}350_{\mathbf{3}}(\sim 700)_{\mathbf{6}}[21] \\
385_{\mathbf{3}}(\sim 700)_{\mathbf{6}}[21] \\
350_{\mathbf{3}}(\sim 700)_{\mathbf{6}}[21]\end{array}\right)$ \\
\hline & 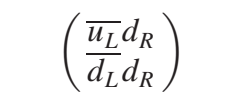 & $\left(\begin{array}{c}(\sim 700)_{\mathbf{8}}[21] \\
(\sim 700)_{8}[21]\end{array}\right)$ & $\left(\begin{array}{l}(\sim 700)_{\mathbf{8}}[21] \\
(\sim 700)_{\mathbf{8}}[21]\end{array}\right)$ \\
\hline$D Q_{1 / 2}$ & $\left(\begin{array}{c}\overline{u_{R}} d_{L} \\
-\overline{u_{R}} u_{L}\end{array}\right)$ & $\left(\begin{array}{l}(\sim 700)_{\mathbf{8}}[21] \\
(\sim 700)_{\mathbf{8}}[21]\end{array}\right)$ & $\left(\begin{array}{c}(\sim 700)_{\mathbf{8}}[21] \\
650_{\mathbf{8}}[22](\sim 700)_{\mathbf{8}}[21]\end{array}\right)$ \\
\hline
\end{tabular}

can be readily obtained by considering the pseudoscalar Yukawa couplings instead of scalar ones. The number of new vectorlike fermion multiplets is less important to the perturbativity bounds, so that many flavors of the new fermions can enhance the diphoton cross section in contrast to the scalar extensions. Because of the above two reasons, heavy vectorlike fermions are sufficient to explain the LHC diphoton excesses.

In conclusion, interpretations of the new exciting results by simple scenarios are the first step for understanding the beyond the SM. As a next step, the requirements of the theoretical consistencies such as the stability and the perturbativity are good criteria for constructing a consistent theory.

\section{ACKNOWLEDGMENTS}

This work is supported by the Grant-in-Aid for Japan Society for the Promotion of Science (JSPS) Fellows No. 25.1107 (Y. H.) and No. 27.1771 (K. K.). K. T.'s work is supported in part by the MEXT Grant-in-Aid for Scientific Research on Innovative Areas No. 26104704.

Note added.-Recently, we became aware of that Ref. [20] appeared on the arXiv, which partially overlaps with our work.

\section{APPENDIX A: LOWER MASS BOUNDS FOR NEW PARTICLES}

In this Appendix, we summarize the direct search bounds on the masses of the new scalar boson and vectorlike fermions with $N_{f}=1$.

In Table VI, collider search bounds for the scalar diquarks are listed. The quantum charges of diquarks in the first column are defined in Table I. The second column presents possible decay modes to the SM particles. The experimental mass bounds strongly depend on the structure of the Yukawa matrices. However, there is no way to specify the structure of the Yukawa matrices without additional assumptions, so that we focus on two classes of the diquarks: (1) diquarks which only decay to the first two generation quarks, (2) diquarks which only decay to the third generation quarks. We here do not consider mixture scenarios for simplicity. Note that if the diquark decays into the third generation quarks, bottom- or top-tagging methods can be applied in order to enhance signal efficiencies.

The scalar diquarks can be produced from the $q q$ fusion if the Yukawa coupling is sufficiently large. Since the bounds are negligible for the smaller Yukawa coupling, we here only take into account the bounds from pair production through the QCD interaction. The lower mass bound for the color triplet diquarks can be estimated from the squark pair production process in the supersymmetric standard model with the R-parity violation [21]. With (without) the b-tagging, the lower mass bounds is $385(350) \mathrm{GeV}$. For color sextet (octet) diquarks, the production cross section is roughly 12.5 (13.5) times larger than that for color triplets. Then, we can read the lower mass bounds for the color sextet and the octet to be about $700 \mathrm{GeV}$. Dedicated searches for the color octet scalar are found in Refs. [22] and [23], which give a weaker bound, $650 \mathrm{GeV}$, as compared to the scaled ones.

In Table VII, we collect the direct search bounds for the scalar leptoquarks. The charge assignments are given in Table II. Flavor changing Yukawa interactions of the leptoquarks are severely constrained by nonobservation of lepton flavor violation (LFV) in low energy data [24]. Therefore, we classify the leptoquarks by the SM fermion generation. ${ }^{12}$ Apart from LFV constraints, masses of

\footnotetext{
${ }^{12} \mathrm{~A}$ leptoquark which couples to one lepton flavor and one quark flavor can avoid stringent bounds from the LFV.
} 
TABLE VII. Lower mass bounds for the leptoquark multiplets.

\begin{tabular}{|c|c|c|c|c|}
\hline & Decay modes & $M_{1 \mathrm{st}} / \mathrm{GeV} \gtrsim$ & $M_{2 \mathrm{nd}} / \mathrm{GeV} \gtrsim$ & $M_{3 \mathrm{rd}} / \mathrm{GeV} \gtrsim$ \\
\hline \multirow{2}{*}{$S_{0}^{d *}$} & $\overline{u_{L}^{c}} e_{L}-\overline{d_{L}^{c}} \nu_{L}$ & $850[25]$ & $760[25]$ & $560[26]$ \\
\hline & $\overline{u_{R}^{c}} e_{R}$ & $1100[25]$ & $1080[25]$ & $685[26]$ \\
\hline$S_{0}^{y *}$ & $\overline{d_{R}^{c}} e_{R}$ & $1100[25]$ & $1080[25]$ & $740[27]$ \\
\hline$S_{1}^{*}$ & $\left(\begin{array}{c}-\overline{u_{L}^{c}} e_{L}^{c} e_{L} \\
\frac{u_{L}^{c}}{u_{L}^{c}} \nu_{L}\end{array}\right.$ & $\left(\begin{array}{c}1100[25] \\
850[25] \\
440[31]\end{array}\right)$ & $\left(\begin{array}{c}1080[25] \\
760[25] \\
540[33]\end{array}\right)$ & $\left(\begin{array}{l}740[28] \\
560[30] \\
750[35]\end{array}\right)$ \\
\hline \multirow{2}{*}{$S_{1 / 2}$} & $\left(\begin{array}{l}\overline{u_{L}} e_{R} \\
\overline{d_{L}} e_{R}\end{array}\right)$ & $\left(\begin{array}{l}1100[25] \\
1100[25]\end{array}\right)$ & $\left(\begin{array}{l}1080[25] \\
1080[25]\end{array}\right)$ & $\left(\begin{array}{l}685[30] \\
740[28]\end{array}\right)$ \\
\hline & $\left(\begin{array}{c}\overline{u_{R}} e_{L} \\
-\overline{u_{R}} \nu_{L}\end{array}\right)$ & $\left(\begin{array}{c}1100[25] \\
440[31]\end{array}\right)$ & $\left(\begin{array}{c}1080[25] \\
540[33]\end{array}\right)$ & $\left(\begin{array}{l}685[30] \\
750[35]\end{array}\right)$ \\
\hline$S_{1 / 2}^{q}$ & $\left(\begin{array}{c}\overline{d_{R}} e_{L} \\
-\overline{d_{R}} \nu_{L}\end{array}\right)$ & $\left(\begin{array}{c}1100[25] \\
440[31]\end{array}\right)$ & $\left(\begin{array}{c}1080[25] \\
440[31]\end{array}\right)$ & $\left(\begin{array}{l}740[28] \\
700[34]\end{array}\right)$ \\
\hline$R_{0}^{u *}$ & $\overline{u_{R}^{c}} \nu_{R}$ & $440[28]$ & 540 [29] & $750[30]$ \\
\hline$R_{0}^{d *}$ & $\overline{d_{R}^{c}} \nu_{R}$ & $440[28]$ & $440[28]$ & $700[31]$ \\
\hline$R_{1 / 2}^{*}$ & $\left(\begin{array}{c}\overline{\nu_{R}} d_{L} \\
-\overline{\nu_{R}} u_{L}\end{array}\right)$ & $\left(\begin{array}{l}440[31] \\
440[31]\end{array}\right)$ & $\left(\begin{array}{l}440[31] \\
540[33]\end{array}\right)$ & $\left(\begin{array}{l}700[34] \\
750[35]\end{array}\right)$ \\
\hline
\end{tabular}

leptoquarks are also strongly constrained by direct searches at the LHC, because of large enough production cross sections by strong interaction and clean leptonic signatures. Even if the decay products contain neutrinos, missing energies play an important role as a trigger.

Bounds for the first two generation leptoquarks, which have a decay mode containing a charged lepton, are presented in Refs. [25,32]. These constraints come from the combination of $\ell \ell j j$ and $\ell \nu j j$ final states in the pair production of a color triplet scalar. There is another constraint from a single production through the Yukawa coupling [33], which can be easily evaded by assuming small Yukawa couplings. For the third generation leptoquarks, similar bounds are found in Refs. [26,27,34]. Although, tau leptons are difficult to identify in the detectors due to the missing neutrinos in their decay chains, further selection cuts such as $b$ - or $t$ - tagging make the bounds stronger. The bounds for the leptoquark related to $\nu_{R}$ can be estimated from the squark pair production in the massless neutralino limit. For the first two generation, $M_{\phi}>440(540) \mathrm{GeV}$ is obtained [28,35] (the charm-tagging technique is applied in Ref. [29]). For the third generation associated with the bottom (top) quark, $M_{\phi}>700$ (750) $\mathrm{GeV}$ is provided in Ref. [31] ([30]).

In Table VIII, direct search bounds for the scalar dileptons are summarized. The representations under the

TABLE VIII. Lower mass bounds for the dilepton multiplets. For the singly charged singlet, more than 2 flavors are required in order to have the Yukawa couplings. The numbers in the parenthesis indicate the existence of other decay modes, which can weaken the lower mass bounds.

\begin{tabular}{|c|c|c|c|c|}
\hline & Decay modes & $M_{1 \mathrm{st}} / \mathrm{GeV} \gtrsim$ & $M_{2 \text { nd }} / \mathrm{GeV} \gtrsim$ & $M_{3 \mathrm{rd}} / \mathrm{GeV} \gtrsim$ \\
\hline$h_{0}^{+}$ & $\overline{\nu_{L i}^{c}} e_{L j}-\overline{e_{L i}^{c}} \nu_{L j} \overline{e_{R}^{c}} \nu_{R}$ & $\left(\begin{array}{c}\text { N.A. } \\
200[36]\end{array}\right)$ & $\left(\begin{array}{c}\text { N.A. } \\
210[36]\end{array}\right)$ & $\left(\begin{array}{c}\text { N.A. } \\
90.6[38]\end{array}\right)$ \\
\hline$h_{0}^{++}$ & $\overline{e_{R}^{c}} e_{R}$ & $374[36]$ & $438[36]$ & $169[37]$ \\
\hline$\Delta_{1}=$ & $\left(\begin{array}{c}-\overline{e_{L}^{c}} e_{L} \\
-\overline{\nu_{L}^{c}} \frac{e_{L}}{\overline{e_{L}^{c}}}-\overline{e_{L}^{c}} \nu_{L} \\
\overline{\nu_{L}^{c}} \nu_{L}\end{array}\right)$ & $\left(\begin{array}{c}(551)[40] \\
(200)[36] \\
\sim M_{Z} / 2\end{array}\right)$ & $\left(\begin{array}{c}(516)[40] \\
(210)[36] \\
\sim M_{Z} / 2\end{array}\right)$ & $\left(\begin{array}{c}(204)[42] \\
(90.6)[38] \\
\sim M_{Z} / 2\end{array}\right)$ \\
\hline & $\left(\begin{array}{c}\overline{\nu_{L}} e_{R} \\
\overline{e_{L}} e_{R}\end{array}\right)$ & $\left(\begin{array}{c}(270)[36] \\
\sim M_{Z} / 2\end{array}\right)$ & $\left(\begin{array}{c}(270)[36] \\
\sim M_{Z} / 2\end{array}\right)$ & $\left(\begin{array}{c}(94)[39] \\
\sim M_{Z} / 2\end{array}\right)$ \\
\hline$\Phi_{1 / 2}$ & $\left(\begin{array}{c}\overline{\nu_{R}} e_{L} \\
-\overline{\nu_{R}} \nu_{L}\end{array}\right)$ & $\left(\begin{array}{c}(270)[36] \\
\sim M_{Z} / 2\end{array}\right)$ & $\left(\begin{array}{c}(270)[36] \\
\sim M_{Z} / 2\end{array}\right)$ & $\left(\begin{array}{c}(94)[39] \\
\sim M_{Z} / 2\end{array}\right)$ \\
\hline$s_{0}$ & $\overline{\nu_{R}^{c}} \nu_{R}$ & $\cdots$ & $\cdots$ & $\cdots$ \\
\hline
\end{tabular}


SM gauge group are given in Table III. In the second column, we list the possible Yukawa interaction with the SM leptons and $\nu_{R}$. The LHC bounds for scalar dileptons are generally loose since its production mechanism relies on the weak interaction. Unlike colored scalar multiplets, dileptons can decay to a pair of SM bosons, $W / Z$ and $h_{125}$. Such decay modes can weaken the direct search bounds on dilepton masses. Furthermore, the mass bounds for an extra doublet $\Phi$ strongly depend on the other Yukawa couplings. The numbers in the parenthesis indicate the presence of such additional decay modes, which may weaken the lower mass bounds. Several dilepton fields are sometimes discussed in the models for small neutrino masses. In such a case, the structure of the Yukawa interactions are constrained by the model. We here classify the dileptons by the SM lepton generation for simplicity.

A bound for singly charged scalar bosons is read from the slepton mass bounds in massless neutralino limit [38-40]. Note that the gauge couplings of singly charged Higgs bosons in $h_{0}^{ \pm}$and $\Delta_{1}$ are the same as those of the right-handed sleptons. The singly charged scalar bosons in the doublet have the same charges with the left-handed slepton. In the Type-II two Higgs doublet model assuming $\mathcal{B}(\tau \nu+c s)=1$, a weaker bound for a singly charged scalar is given by LEP [41]. The bounds for the neutral component in the doublet have large ambiguity due to the possible Yukawa coupling to the quarks. We have, at least, a bound of $\sim M_{Z} / 2$ from the precisely measured $Z$ boson decay width. Potentially strong bounds are found for doubly charged scalar bosons by assuming the same-sign two lepton decay modes $[36,42]$. If the doubly charged scalars decay to a pair of $W$ boson (it is possible for the triplet dilepton case), the lower mass bound is only $84 \mathrm{GeV}$ [43] from the inclusive search mode. No bound is obtained for the SM singlet scalar $s_{0}$.

In Table IX, lower mass bounds for the third generation vectorlike quarks are listed. The quantum charges of them are defined in Table IV. The second column is a guideline for estimating the decay branching ratios of vectorlike fermions, where the Higgs field is expanded as $\Phi=\left(i G^{+}, \frac{H-i G^{0}}{\sqrt{2}}\right)^{T}$. The NG boson fields $G^{0}, G^{ \pm}$indicate the decays to $Z, W$ bosons in the heavy mass limit through the electroweak equivalence theorem.

Thanks to the bottom- and/or top-tagging technique, stronger lower bounds of $735-855 \mathrm{GeV}$ are obtained for the vector-like bottom $\left(Q_{\psi}=-1 / 3\right)$ and top quarks $\left(Q_{\psi}=2 / 3\right)$ depending on the branching fractions [44,45]. Slightly stronger bounds of $890-920 \mathrm{GeV}$ are given for the vectorlike fermions with the charge $5 / 3$ and $-4 / 3$, since the flipped charged $W$ boson is a good discriminant from the background events as compared with the conventional vectorlike top and bottom quark decays to the $W$ boson $[46,47]$. If a vectorlike quark decays into the a light quark (the first two generations), the bounds become weaker.
TABLE IX. Lower mass bounds for the vectorlike quark multiplets.

\begin{tabular}{lcc}
\hline \hline & Decay modes & $M_{3 \mathrm{rd}} / \mathrm{GeV} \gtrsim$ \\
\hline$T_{0}$ & $\overline{t_{L}} \frac{H+i G^{0}}{\sqrt{2}}+\overline{b_{L}} i G^{-}$ & $800[44]$ \\
$B_{0}$ & $\overline{t_{L}} i G^{+}+\overline{b_{L}} \frac{H-i G^{0}}{\sqrt{2}}$ & $735[44]$ \\
$T_{1}$ & $\left(\begin{array}{c}\overline{t_{L}} i G^{-} \\
\overline{t_{L}} \frac{H+i G^{0}}{\sqrt{2}}+\overline{b_{L}} i G^{-} \\
\overline{b_{L}} \frac{H+i G^{0}}{\sqrt{2}}\end{array}\right)$ & $\left(\begin{array}{l}890[46] \\
800[44] \\
755[45]\end{array}\right)$ \\
$B_{1}$ & $\left(\begin{array}{c}\overline{t_{L}} \frac{H-i G^{0}}{\sqrt{2}} \\
\overline{t_{L}} i G^{+}+\overline{b_{L}} \frac{H-i G^{0}}{\sqrt{2}} \\
\overline{b_{L}} i G^{+}\end{array}\right)$ & $\left(\begin{array}{l}855[44] \\
735[44] \\
920[47]\end{array}\right)$ \\
$Q_{1 / 2}$ & $\left(\begin{array}{l}\overline{t_{R}} \frac{H-i G^{0}}{\sqrt{2}} \\
-\overline{t_{R}} i G^{+}\end{array}\right)$ & $\left(\begin{array}{l}855[44] \\
890[46]\end{array}\right)$ \\
$T_{1 / 2}$ & $\left(\begin{array}{c}\overline{b_{R}} i G^{-} \\
-\overline{b_{R}} \frac{H+i G^{0}}{\sqrt{2}}\end{array}\right)$ & $\left(\begin{array}{l}920[47] \\
755[45]\end{array}\right)$ \\
$B_{1 / 2}$ & $\left(\begin{array}{c}\overline{t_{R}} i G^{-} \\
-\overline{t_{R}} \frac{H+i G^{0}}{\sqrt{2}}\end{array}\right)$ & $\left(\begin{array}{l}890[46] \\
855[44]\end{array}\right)$ \\
\hline \hline & $\left(\begin{array}{l}b_{R} \\
-\overline{b_{R}} i-i G^{0} \\
\overline{2}\end{array}\right)$ & $\left(\begin{array}{l}755[45] \\
920[47]\end{array}\right)$ \\
\hline
\end{tabular}

For example, a vectorlike quark of $\mathcal{B}(W q)=1(0.5)$ is excluded from 320(390) $\mathrm{GeV}$ to 690(410) $\mathrm{GeV}$ [48].

In Table X, lower mass bounds for the vectorlike leptons are summarized. The charge assignments are given in

TABLE X. Lower mass bounds for the vectorlike lepton multiplets.

\begin{tabular}{|c|c|c|c|}
\hline & Decay modes & $M_{1 \mathrm{st}} / \mathrm{GeV} \gtrsim$ & $M_{2 \mathrm{st}} / \mathrm{GeV} \gtrsim$ \\
\hline$N_{0}$ & $\overline{\nu_{L}} \frac{H+i G^{0}}{\sqrt{2}}+\overline{e_{L}} i G^{-}$ & $\cdots$ & $\ldots$ \\
\hline$E_{0}$ & $\overline{\nu_{L}} i G^{+}+\overline{e_{L}} \frac{H-i G^{0}}{\sqrt{2}}$ & $176^{\mathrm{a}}[49]$ & $168^{\mathrm{b}}[49]$ \\
\hline$N_{1}$ & $\left(\begin{array}{c}\overline{\nu_{L}} i G^{-} \\
\overline{\nu_{L}} \frac{H+i G^{0}}{\sqrt{2}}+\overline{e_{L}} i G^{-} \\
\overline{e_{L}} \frac{H+i G^{0}}{\sqrt{2}}\end{array}\right)$ & 430 [49] & $468^{c}$ [49] \\
\hline$E_{1}$ & $\begin{array}{c}\overline{\nu_{L}} \frac{H-i G^{0}}{\sqrt{2}} \\
\overline{\nu_{L}} i G^{+}+\overline{e_{L}} \frac{H-i G^{0}}{\sqrt{2}} \\
\overline{e_{I}} i G^{+}\end{array}$ & $\left(\begin{array}{c}\sim M_{Z} / 2 \\
176^{\mathrm{a}}[49] \\
\sim M_{Z} / 2\end{array}\right)$ & $\left(\begin{array}{c}\sim M_{Z} / 2 \\
168^{\mathrm{b}}[49] \\
\sim M_{Z} / 2\end{array}\right)$ \\
\hline & $\left(\begin{array}{c}\overline{\nu_{R}} \frac{H-i G^{0}}{\sqrt{2}} \\
-\overline{\nu_{R}} i G^{+}\end{array}\right)$ & $\left(\begin{array}{c}300[52] \\
101.2[53]\end{array}\right)$ & $\left(\begin{array}{c}300[52] \\
101.2[53]\end{array}\right)$ \\
\hline$L_{1 / 2}$ & $\left(\begin{array}{c}\overline{\nu_{R}^{c}} \frac{H-i G^{0}}{\sqrt{2}} \\
-\overline{\nu_{R}^{c}} i G^{+}\end{array}\right)$ & $\left(\begin{array}{c}300[52] \\
101.2[53]\end{array}\right)$ & $\left(\begin{array}{c}300[52] \\
101.2[53]\end{array}\right)$ \\
\hline & $\left(\begin{array}{c}\overline{e_{R}} i G^{-} \\
-\overline{e_{R}} \frac{H+i G^{0}}{\sqrt{2}}\end{array}\right)$ & $\left(\begin{array}{c}102.6[53] \\
\sim M_{Z} / 2\end{array}\right)$ & $\left(\begin{array}{c}102.7[53] \\
\sim M_{Z} / 2\end{array}\right)$ \\
\hline$E_{1 / 2}$ & $\left(\begin{array}{c}\overline{e_{R}} \frac{H-i G^{0}}{\sqrt{2}} \\
-\overline{e_{R}} i G^{+}\end{array}\right)$ & $\left(\begin{array}{l}\sim M_{Z} / 2 \\
\sim M_{Z} / 2\end{array}\right)$ & $\left(\begin{array}{l}\sim M_{Z} / 2 \\
\sim M_{Z} / 2\end{array}\right)$ \\
\hline
\end{tabular}

\footnotetext{
${ }^{\mathrm{a}}$ Except for $<129 \mathrm{GeV}$ and $144-163 \mathrm{GeV}$.

${ }^{\mathrm{b}}$ Except for $<144 \mathrm{GeV}$ and $153-160 \mathrm{GeV}$.

${ }^{c}$ Except for $401-419 \mathrm{GeV}$.
} 
Table V. Since the electrons and muons can be a clean signal at hadron colliders, the vectorlike leptons that couple to the first two generation have been searched. If the vectorlike lepton only decays to tau lepton modes, weaker experimental bounds are expected.

The triplet vectorlike lepton $N_{1}$, which appears in Type-III seesaw model, is analyzed by assuming the degeneracy among different charge components. The bound is relatively strong because of the large cross section through the $s$-channel $W$ exchange diagram [49]. The bound on the singlet vectorlike lepton $E_{0}$ is weaker, since it is produced only in a pair via $Z$ bosons and photons (the production cross section is much smaller than that of triplet vectorlike leptons via $W$ bosons). This result is also applicable for the singly charged component of $E_{1}$. A bound for the Higgsino decaying to $H$ or $Z$ with a gravitino can be reinterpreted for the bound on the neutral component of $L_{1 / 2}$ [50]. Assuming the 50\% branching fractions both for $H$ and $Z$ modes, a lower mass bound of $300 \mathrm{GeV}$ is estimated. The bound for the charged component comes from the LEP direct search [51].

\section{APPENDIX B: RENORMALIZATION GROUP EQUATIONS}

Here, we give the one-loop RGEs of our models. First, the one-loop RGEs of the SM gauge couplings are as follows:

$$
\begin{aligned}
\frac{d g_{Y}}{d t} & =\frac{g_{Y}^{3}}{(4 \pi)^{2}}\left(\frac{41}{6}+N_{f} N_{\psi} n_{\psi} \frac{4}{3} Y_{\psi}^{2}+N_{f} N_{\phi} n_{\phi} \frac{1}{3} Y_{\phi}^{2}\right), \\
\frac{d g_{2}}{d t} & =\frac{g_{2}^{3}}{(4 \pi)^{2}}\left(-\frac{19}{6}+N_{f} N_{\psi} \frac{4}{3} S_{2}(F)+N_{f} N_{\phi} \frac{1}{3} S_{2}(S)\right), \\
\frac{d g_{3}}{d t} & =\frac{g_{3}^{3}}{(4 \pi)^{2}}\left(-7+N_{f} n_{\psi} \frac{4}{3} S_{2}^{(c)}(F)+N_{f} n_{\phi} \frac{1}{3} S_{2}^{(c)}(S)\right) .
\end{aligned}
$$

Here, $t:=\log \mu$ with $\mu$ being the renormalization scale, $Y_{\phi}\left(Y_{\psi}\right)$ is the $U(1)_{Y}$ hypercharge of $\phi(\psi), n_{\phi}\left(N_{\psi}\right)$ is the $S U(2)_{L}$ representation of $\phi(\psi), N_{\phi}\left(N_{\psi}\right)$ is the $S U(3)_{c}$ representation of $\phi(\psi), S_{2}(S)\left(S_{2}(F)\right)$ is the $S U(2){ }_{L}$ Dynkin index of $\phi(\psi)$, and $S_{2}^{(c)}(S)\left(S_{2}^{(c)}(F)\right)$ is the $S U(3)_{c}$ Dynkin index of $\phi(\psi)$.

Next, we summarize the one-loop RGEs of the scalar quartic couplings for each scalar model. Note that it is convenient to use the Fierz identity in order to see the number of independent terms in the scalar potential, Eq. (5). The Fierz identities are given by

$$
\begin{aligned}
\left(t^{a}\right)_{j}^{i}\left(t^{a}\right)_{l}^{k} & =\frac{1}{2}\left(-\frac{1}{2} \delta_{j}^{i} \delta_{l}^{k}+\delta_{l}^{i} \delta_{j}^{k}\right), \\
\left\{t^{a}, t^{b}\right\}_{j}^{i}\left\{t^{a}, t^{b}\right\}_{l}^{k} & =\frac{3}{4} \delta_{j}^{i} \delta_{l}^{k}, \quad \text { for } S U(2) \mathbf{2}, \\
\left\{t^{a}, t^{b}\right\}_{j}^{i}\left\{t^{a}, t^{b}\right\}_{l}^{k} & =A \delta_{j}^{i} \delta_{l}^{k}+(8-A) \delta_{l}^{i} \delta_{j}^{k}+(-6+A)\left(t^{a}\right)_{j}^{i}\left(t^{a}\right)_{l}^{k}+(4-A)\left(t^{a}\right)_{l}^{i}\left(t^{a}\right)_{j}^{k}, \quad \text { for } S U(2) \mathbf{3}, \\
\left(T^{a}\right)_{j}^{i}\left(T^{a}\right)_{l}^{k} & =\frac{1}{2}\left(-\frac{1}{3} \delta_{j}^{i} \delta_{l}^{k}+\delta_{l}^{i} \delta_{j}^{k}\right), \\
\left\{T^{a}, T^{b}\right\}_{j}^{i}\left\{T^{a}, T^{b}\right\}_{l}^{k} & =\frac{11}{18} \delta_{j}^{i} \delta_{l}^{k}+\frac{5}{6} \delta_{l}^{i} \delta_{j}^{k} \quad \text { for } S U(3) \mathbf{3}, \\
\left\{T^{a}, T^{b}\right\}_{j}^{i}\left\{T^{a}, T^{b}\right\}_{l}^{k} & =A \delta_{j}^{i} \delta_{l}^{k}+\left(\frac{80}{9}-A\right) \delta_{l}^{i} \delta_{j}^{k}+\left(-\frac{17}{3}+\frac{3}{2} A\right)\left(T^{a}\right)_{j}^{i}\left(T^{a}\right)_{l}^{k}+\left(\frac{22}{3}-\frac{3}{2} A\right)\left(T^{a}\right)_{l}^{i}\left(T^{a}\right)_{j}^{k}, \quad \text { for } S U(3) \mathbf{6}, \\
\left\{T^{a}, T^{b}\right\}_{j}^{i}\left\{T^{a}, T^{b}\right\}_{l}^{k} & =3 \delta_{j}^{i} \delta_{l}^{k}+3 \delta_{l}^{i} \delta_{j}^{k}+3 \delta_{k}^{i} \delta_{l}^{j}-\left(T^{a}\right)_{j}^{i}\left(T^{a}\right)_{l}^{k}+2\left(T^{a}\right)_{l}^{i}\left(T^{a}\right)_{j}^{k}, \quad \text { for } S U(3) \mathbf{8}, \\
\operatorname{Tr}\left(T^{a} T^{b} T^{c} T^{d}\right) & =\frac{3}{4} \delta^{a b} \delta^{c d}+\frac{3}{4} \delta^{b c} \delta^{a d}+\frac{3}{4} \delta^{a c} \delta^{b d}+\frac{1}{2}\left(T^{e}\right)_{c}^{a}\left(T^{e}\right)_{d}^{b}+\left(T^{e}\right)_{d}^{a}\left(T^{e}\right)_{c}^{b}, \quad \text { for } S U(3) \mathbf{8},
\end{aligned}
$$

where $A$ is a free parameter. Among the various scalar quartic couplings, we can write the beta functions of $\lambda, \lambda_{X}, \kappa_{H \phi}, \kappa_{H \phi}^{\prime}$, $\kappa_{H X}, \kappa_{\phi X}$ generally as follows ${ }^{13}$ :

\footnotetext{
${ }^{13}$ Regarding the terms including $\lambda_{\mathrm{tr}}$ and $\lambda_{\mathrm{ad}}^{\prime}$, we assume $N_{f}=1$.
} 
MODELS OF THE LHC DIPHOTON EXCESSES VALID UP ...

$$
\begin{aligned}
\frac{d \lambda}{d t}= & \frac{1}{16 \pi^{2}}\left(\frac{1}{4} S_{2}(S) N_{\phi} N_{f} \kappa_{H \phi}^{\prime 2}+n_{\phi} N_{\phi} N_{f} \kappa_{H \phi}^{2}-9 g_{2}^{2} \lambda-3 \lambda g_{Y}^{2}\right. \\
& \left.+\frac{3}{4} g_{2}^{2} g_{Y}^{2}+\frac{3 g_{Y}^{4}}{8}+\frac{9 g_{2}^{4}}{8}+\frac{\kappa_{\mathrm{HX}}^{2}}{2}+24 \lambda^{2}+12 \lambda y_{t}^{2}-6 y_{t}^{4}\right) \\
\frac{d \lambda_{X}}{d t}= & \frac{1}{16 \pi^{2}}\left(3 \lambda_{X}^{2}+12 \kappa_{H X}^{2}+6 n_{\phi} N_{\phi} N_{f} \kappa_{\phi X}^{2}\right), \\
\frac{d \kappa_{H \phi}}{d t}= & \frac{1}{16 \pi^{2}}\left(6 y_{t}^{2} \kappa_{H \phi}+4 \kappa_{H \phi}^{2}+C_{2}(S) \kappa_{H \phi}^{\prime 2}+12 \kappa_{H \phi} \lambda+\kappa_{\phi X} \kappa_{H X}+4 C_{2}(S) \kappa_{H \phi} \lambda_{\phi}^{\prime \prime}+4 C_{2}^{(c)}(S) \kappa_{H \phi} \lambda_{\phi}^{\prime}\right. \\
& +4 C_{2}(S) C_{2}^{(c)}(S) \kappa_{H \phi} \lambda_{\phi}^{\prime \prime \prime}+\left(4 N_{f} n_{\phi} N_{\phi}+4\right) \kappa_{H \phi} \lambda_{\phi}+4\left(n_{\phi}+N_{\phi}\right) \kappa_{H \phi} \lambda_{\mathrm{tr}}+3 Y_{\phi}^{2} g_{Y}^{4}+3 C_{2}(S) g_{2}^{4} \\
& \left.-\left(\frac{9}{2}+6 C_{2}(S)\right) \kappa_{H \phi} g_{2}^{2}-\left(\frac{3}{2}+6 Y_{\phi}^{2}\right) \kappa_{H \phi} g_{Y}^{2}-6 C_{2}^{(c)}(S) \kappa_{H \phi} g_{3}^{2}+4\left(n_{\phi}+1\right) \kappa_{H \phi} \lambda_{\mathrm{ad}}^{\prime}\right), \\
\frac{d \kappa_{H \phi}^{\prime}}{d t}= & \frac{1}{16 \pi^{2}}\left(6 y_{t}^{2} \kappa_{H \phi}^{\prime}+8 \kappa_{H \phi} \kappa_{H \phi}^{\prime}+4 \kappa_{H \phi}^{\prime} \lambda+4 \kappa_{H \phi}^{\prime} \lambda_{\phi}+4\left(C_{2}(S)-1+N_{\phi} S_{2}(S) N_{f}\right) \kappa_{H \phi}^{\prime} \lambda_{\phi}^{\prime \prime}\right. \\
& +4 C_{2}^{(c)}(S) \kappa_{H \phi}^{\prime} \lambda_{\phi}^{\prime}+4\left(C_{2}(S)-1\right) C_{2}^{(c)}(S) \kappa_{H \phi}^{\prime} \lambda_{\phi}^{\prime \prime \prime}+4 N_{\phi} \kappa_{H \phi}^{\prime} \lambda_{\mathrm{tr}}+4 \kappa_{H \phi}^{\prime} \lambda_{\mathrm{ad}}^{\prime} \\
& \left.-\left(\frac{9}{2}+6 C_{2}(S)\right) \kappa_{H \phi}^{\prime} g_{2}^{2}-\left(\frac{3}{2}+6 Y_{\phi}^{2}\right) \kappa_{H \phi}^{\prime} g_{Y}^{2}-6 C_{2}^{(c)}(S) \kappa_{H \phi}^{\prime} g_{3}^{2}+12 Y_{\phi} g_{Y}^{2} g_{2}^{2}\right), \\
\frac{d \kappa_{H X}}{d t}= & \frac{1}{16 \pi^{2}}\left(\kappa_{H X}\left(12 \lambda+\lambda_{X}+4 \kappa_{H X}+6 y_{t}^{2}-\frac{3}{2} g_{Y}^{2}-\frac{9}{2} g_{2}^{2}\right)+2 n_{\phi} N_{\phi} N_{f} \kappa_{\phi H} \kappa_{\phi X}\right), \\
\frac{d \kappa_{\phi X}}{d t}= & \frac{1}{16 \pi^{2}}\left(4 \kappa_{\phi X}^{2}+\kappa_{\phi X} \lambda_{X}+4 \kappa_{H X} \kappa_{H \phi}+4 C_{2}(S) \kappa_{\phi X} \lambda_{\phi}^{\prime \prime}+4 C_{2}^{(c)}(S) \kappa_{\phi X} \lambda_{\phi}^{\prime}+4 C_{2}(S) C_{2}^{(c)}(S) \kappa_{\phi X} \lambda_{\phi}^{\prime \prime \prime}\right. \\
& +\left(4 N_{f} n_{\phi} N_{\phi}+4\right) \kappa_{\phi X} \lambda_{\phi}+4\left(n_{\phi}+N_{\phi}\right) \kappa_{\phi X} \lambda_{\mathrm{tr}}-6 C_{2}(S) \kappa_{\phi X} g_{2}^{2}-6 Y_{\phi}^{2} \kappa_{\phi X} g_{Y}^{2}-6 C_{2}^{(c)}(S) \kappa_{\phi X} g_{3}^{2} \\
& \left.+4\left(n_{\phi}+1\right) \kappa_{\phi X} \lambda_{\mathrm{ad}}^{\prime}\right) . \\
& \\
&
\end{aligned}
$$

In the following, we show the RGEs of other scalar quartic couplings for each scalar model. Notice that we neglect such a coupling that is not induced at one loop level if we put it zero at the weak scale, and that the $U(1)_{Y}$ hypercharge $Y_{\phi}$ is kept as a free parameter.

(i) $\left(\mathbf{1}, \mathbf{1}, Y_{\phi}\right)$

$$
\frac{d \lambda_{\phi}}{d t}=\frac{1}{16 \pi^{2}}\left(-12 \lambda_{\phi} g_{Y}^{2} Y_{\phi}^{2}+\left(4 N_{f}+16\right) \lambda_{\phi}^{2}+\frac{\kappa_{\phi X}^{2}}{2}+6 g_{Y}^{4} Y_{\phi}^{4}+2 \kappa_{H \phi}^{2}\right)
$$

(ii) $\left(\mathbf{1}, \mathbf{2}, Y_{\phi}\right)^{14}$

$$
\begin{aligned}
\frac{d \lambda_{\phi}}{d t}= & \frac{1}{16 \pi^{2}}\left(-12 \lambda_{\phi} g_{Y}^{2} Y_{\phi}^{2}-9 g_{2}^{2} \lambda_{\phi}+\left(8 N_{f}+16\right) \lambda_{\phi}^{2}+\frac{\kappa_{\phi X}^{2}}{2}+\frac{3}{2} \lambda_{\phi}^{\prime \prime 2}+6 \lambda_{\phi} \lambda_{\phi}^{\prime \prime}+3 g_{2}^{2} g_{Y}^{2} Y_{\phi}^{2} \delta_{N_{f}, 1}+6 g_{Y}^{4} Y_{\phi}^{4}\right. \\
& \left.+\frac{9 g_{2}^{4}}{8}+\frac{1}{8} \kappa_{H \phi}^{\prime 2} \delta_{N_{f}, 1}+2 \kappa_{H \phi}^{2}\right), \\
\frac{d \lambda_{\phi}^{\prime \prime}}{d t}= & \frac{1}{16 \pi^{2}}\left(12 Y_{\phi}^{2} g_{Y}^{2} g_{2}^{2}+\frac{1}{2} \kappa_{H \phi}^{\prime 2}+\left(2 N_{f}-2\right) \lambda_{\phi}^{\prime \prime 2}-12 Y_{\phi}^{2} g_{Y}^{2} \lambda_{\phi}^{\prime \prime}-9 g_{2}^{2} \lambda_{\phi}^{\prime \prime}+24 \lambda_{\phi} \lambda_{\phi}^{\prime \prime}\right),
\end{aligned}
$$

\footnotetext{
${ }^{14} \lambda_{\phi}^{\prime \prime}$ term exists only if $N_{f} \geq 2$.
} 
HAMADA, KAWAI, KAWANA, and TSUMURA

(iii) $\left(\mathbf{1}, \mathbf{3}, Y_{\phi}\right)^{15}$

$$
\begin{aligned}
\frac{d \lambda_{\phi 2}^{\prime}}{d t}= & \frac{1}{16 \pi^{2}}\left(-24 g_{2}^{2} \lambda_{\phi 2}^{\prime}-12 Y^{2} g_{Y}^{2} \lambda_{\phi 2}^{\prime}+\frac{3 g_{2}^{4}}{2}+24 \lambda_{\phi} \lambda_{\phi 2}^{\prime}-8 \lambda_{\phi}^{\prime \prime} \lambda_{\phi 2}^{\prime}+2 \lambda^{\prime \prime 2}{ }_{\phi}+\left(16 N_{f}+152\right) \lambda_{\phi 2}^{\prime 2}\right), \\
\frac{d \lambda_{\phi}^{\prime \prime}}{d t}= & \frac{1}{16 \pi^{2}}\left(-24 g_{2}^{2} \lambda^{\prime \prime}{ }_{\phi}-12 g_{Y}^{2} Y_{\phi}^{2} \lambda_{\phi}^{\prime \prime}+\left(8 N_{f}+8-4{\delta_{N_{f}, 1}}_{1}\right) \lambda_{\phi}^{\prime \prime 2}+24 \lambda_{\phi} \lambda^{\prime \prime}{ }_{\phi}+12 g_{2}^{2} g_{Y}^{2} Y_{\phi}^{2}-3 g_{2}^{4} \delta_{N_{f}, 1}\right. \\
& \left.+\frac{1}{2} \kappa_{H \phi}^{\prime 2}+128 \lambda_{\phi 2}^{\prime} \lambda_{\phi}^{\prime \prime}\right), \\
\frac{d \lambda_{\phi}}{d t}= & \frac{1}{16 \pi^{2}}\left(-24 g_{2}^{2} \lambda_{\phi}-12 \lambda_{\phi} g_{Y}^{2} Y_{\phi}^{2}+16 \lambda^{\prime \prime 2}{ }_{\phi} \delta_{N_{f}, 1}+16 \lambda_{\phi} \lambda_{\phi}^{\prime \prime}+\left(12 N_{f}+16\right) \lambda_{\phi}^{2}+\frac{\kappa_{\phi X}^{2}}{2}+6 g_{Y}^{4} Y_{\phi}^{4}+12 g_{2}^{4} \delta_{N_{f}, 1}\right. \\
& \left.+2 \kappa_{H \phi}^{2}+\left(128 N_{f}+96\right) \lambda_{\phi} \lambda_{\phi 2}^{\prime}+256 N_{f} \lambda_{\phi 2}^{\prime 2}+128 \lambda_{\phi}^{\prime \prime} \lambda_{\phi 2}^{\prime}\right),
\end{aligned}
$$

(iv) $\left(\mathbf{3}\left(\mathbf{3}^{*}\right), \mathbf{1}, Y_{\phi}\right)^{16}$

$$
\begin{aligned}
\frac{d \lambda_{\phi}}{d t}= & \frac{1}{16 \pi^{2}}\left(-16 g_{3}^{2} \lambda_{\phi}-12 g_{Y}^{2} \lambda_{\phi} Y_{\phi}^{2}+4 g_{3}^{2} g_{Y}^{2} Y_{\phi}^{2} \delta_{N_{f}, 1}+6 g_{Y}^{4} Y_{\phi}^{4}+\left(\frac{4}{3}+\frac{5}{6} \delta_{N_{f}, 1}\right) g_{3}^{4}+2 \kappa_{H \phi}^{2}+\left(12 N_{f}+16\right) \lambda_{\phi}^{2}\right. \\
& \left.+\frac{\kappa_{\phi X}^{2}}{2}+\frac{32}{3} \lambda_{\phi} \lambda_{\phi}^{\prime}+\frac{16}{9} \lambda_{\phi}^{\prime 2}\right), \\
\frac{d \lambda_{\phi}^{\prime}}{d t}= & \frac{1}{16 \pi^{2}}\left(-16 g_{3}^{2} \lambda_{\phi}^{\prime}-12 g_{Y}^{2} \lambda_{\phi}^{\prime} Y_{\phi}^{2}+12 Y_{\phi}^{2} g_{Y}^{2} g_{3}^{2}+\frac{5}{2} g_{3}^{4}+24 \lambda_{\phi} \lambda_{\phi}^{\prime}+2\left(N_{f}+1\right) \lambda_{\phi}^{\prime 2}\right),
\end{aligned}
$$

(v) $\left(\mathbf{3}\left(\mathbf{3}^{*}\right), \mathbf{2}, Y_{\phi}\right)^{17}$

$$
\begin{aligned}
\frac{d \lambda_{\phi}}{d t}= & \frac{1}{16 \pi^{2}}\left(-9 g_{2}^{2} \lambda_{\phi}-16 g_{3}^{2} \lambda_{\phi}-12 g_{Y}^{2} Y_{\phi}^{2} \lambda_{\phi}+\left(24 N_{f}+16\right) \lambda_{\phi}^{2}-g_{2}^{2} g_{Y}^{2} Y_{\phi}^{2} \delta_{N_{f}, 1}+6 g_{Y}^{4} Y_{\phi}^{4}+\frac{9 g_{2}^{4}}{8}+\frac{8}{3} g_{3}^{2} g_{2}^{2} \delta_{N_{f}, 1}\right. \\
& \left.+\frac{4 g_{3}^{4}}{3}+2 \kappa_{H \phi}^{2}+\frac{\kappa_{\phi X}^{2}}{2}+\frac{32}{3} \lambda_{\phi} \lambda_{\phi}^{\prime}+6 \lambda_{\phi} \lambda_{\phi}^{\prime \prime}+8 \lambda_{\phi} \lambda_{\phi}^{\prime \prime \prime}+\frac{3}{2} \lambda_{\phi}^{\prime \prime 2}+\frac{16}{9} \lambda_{\phi}^{\prime 2}+\frac{1}{3} \lambda_{\phi}^{\prime \prime \prime 2}\right), \\
\frac{d \lambda_{\phi}^{\prime}}{d t}= & \frac{1}{16 \pi^{2}}\left(-16 g_{3}^{2} \lambda_{\phi}^{\prime}-9 g_{2}^{2} \lambda_{\phi}^{\prime}-12 g_{Y}^{2} Y_{\phi}^{2} \lambda_{\phi}^{\prime}+\left(4 N_{f}+2\right) \lambda_{\phi}^{\prime 2}+24 \lambda_{\phi} \lambda_{\phi}^{\prime}+3 \lambda_{\phi}^{\prime \prime} \lambda_{\phi}^{\prime \prime \prime}-\lambda_{\phi} \lambda_{\phi}^{\prime \prime \prime}\right. \\
& \left.+12 g_{3}^{2} g_{Y}^{2} Y_{\phi}^{2}+12 g_{2}^{2} g_{Y}^{2} Y_{\phi}^{2} \delta_{N_{f}, 1}+\frac{5 g_{3}^{4}}{2}-5 g_{2}^{2} g_{3}^{2} \delta_{N_{f}, 1}+\frac{5}{8} \lambda_{\phi}^{\prime \prime \prime 2}+6 \lambda_{\phi}^{\prime} \lambda_{\phi}^{\prime \prime}-\lambda_{\phi}^{\prime} \lambda_{\phi}^{\prime \prime \prime}\right), \\
\frac{d \lambda_{\phi}^{\prime \prime}}{d t}= & \frac{1}{16 \pi^{2}}\left(-16 g_{3}^{2} \lambda_{\phi}^{\prime \prime \prime}-9 g_{2}^{2} \lambda_{\phi}^{\prime \prime}-12 g_{Y}^{2} Y_{\phi}^{2} \lambda_{\phi}^{\prime \prime}+\left(6 N_{f}-2\right) \lambda^{\prime \prime 2}{ }_{\phi}+24 \lambda_{\phi} \lambda_{\phi}^{\prime \prime}+12 g_{2}^{2} g_{Y}^{2} Y_{\phi}^{2}\right. \\
& \left.+\frac{1}{2} \kappa_{H \phi}^{\prime 2}+\frac{32}{3} \lambda_{\phi}^{\prime} \lambda_{\phi}^{\prime \prime}-\frac{8}{3} \lambda_{\phi}^{\prime \prime} \lambda_{\phi}^{\prime \prime \prime}+\frac{32}{9} \lambda_{\phi}^{\prime} \lambda_{\phi}^{\prime \prime \prime}\right), \\
\frac{d \lambda_{\phi}^{\prime \prime \prime}}{d t}= & \frac{1}{16 \pi^{2}}\left(-16 g_{3}^{2} \lambda_{\phi}^{\prime \prime \prime}-9 g_{2}^{2} \lambda_{\phi}^{\prime \prime \prime}-12 g_{Y}^{2} Y_{\phi}^{2} \lambda_{\phi}^{\prime \prime \prime}+12 g_{2}^{2} g_{3}^{2}+\left(N_{f}+\frac{10}{3}\right) \lambda_{\phi}^{\prime \prime \prime 2}+\frac{16}{3} \lambda_{\phi}^{\prime} \lambda_{\phi}^{\prime \prime \prime}+16 \lambda_{\phi}^{\prime} \lambda_{\phi}^{\prime \prime}\right. \\
& \left.+24 \lambda_{\phi} \lambda_{\phi}^{\prime \prime \prime}-2 \lambda_{\phi}^{\prime \prime} \lambda_{\phi}^{\prime \prime \prime}\right),
\end{aligned}
$$

\footnotetext{
${ }^{15}$ Notice that $\lambda_{\phi 2}^{\prime}$ term exists only if $N_{f} \geq 2$, and that $\lambda_{\text {ad }}$ is not induced at one loop level.

${ }^{16} \lambda_{\phi}^{\prime}$ term exists only if $N_{f} \geq 2$.

${ }^{17} \lambda_{\phi}^{\prime \prime}$ and $\lambda_{\phi}^{\prime \prime \prime}$ terms exist only if $N_{f} \geq 2$.
} 


\section{MODELS OF THE LHC DIPHOTON EXCESSES VALID UP ...}

(vi) $\left(\mathbf{3}\left(\mathbf{3}^{*}\right), \mathbf{3}, Y_{\phi}\right), N_{f}=1^{18}$

$$
\begin{aligned}
\frac{d \lambda_{\phi}}{d t}= & \frac{1}{16 \pi^{2}}\left(-24 g_{2}^{2} \lambda_{\phi}-16 g_{3}^{2} \lambda_{\phi}-12 g_{Y}^{2} \lambda_{\phi} Y_{\phi}^{2}+6 g_{Y}^{4} Y_{\phi}^{4}+8 g_{2}^{4}+4 g_{3}^{2} g_{2}^{2}+\frac{4 g_{3}^{4}}{3}+2 \kappa_{H \phi}^{2}+16 \lambda_{\phi} \lambda_{\phi}^{\prime \prime}\right. \\
& \left.+\frac{16}{9} \lambda_{\phi}^{\prime 2}+\frac{32}{3} \lambda_{\phi}^{\prime \prime 2}+\frac{32}{3} \lambda_{\phi} \lambda_{\phi}^{\prime}+\frac{16}{3} \lambda_{\phi}^{\prime} \lambda_{\phi}^{\prime \prime}+52 \lambda_{\phi}^{2}+\frac{\kappa_{\phi X}^{2}}{2}\right), \\
\frac{d \lambda_{\phi}^{\prime}}{d t}= & \frac{1}{16 \pi^{2}}\left(-24 g_{2}^{2} \lambda_{\phi}^{\prime}-16 g_{3}^{2} \lambda_{\phi}^{\prime}-12 g_{Y}^{2} Y_{\phi}^{2} \lambda_{\phi}^{\prime}+12 g_{3}^{2} g_{Y}^{2} Y_{\phi}^{2}+12 g_{2}^{4}-12 g_{3}^{2} g_{2}^{2}+\frac{5 g_{3}^{4}}{2}+8 \lambda_{\phi}^{\prime 2}\right. \\
& \left.+16 \lambda_{\phi}^{\prime \prime 2}+24 \lambda_{\phi} \lambda_{\phi}^{\prime}+16 \lambda_{\phi}^{\prime} \lambda_{\phi}^{\prime \prime}\right), \\
\frac{d \lambda_{\phi}^{\prime \prime}}{d t}= & \frac{1}{16 \pi^{2}}\left(-24 g_{2}^{2} \lambda_{\phi}^{\prime \prime}-16 g_{3}^{2} \lambda_{\phi}^{\prime \prime}-12 g_{Y}^{2} Y_{\phi}^{2} \lambda_{\phi}^{\prime \prime}+12 g_{2}^{2} g_{Y}^{2} Y_{\phi}^{2}-3 g_{2}^{4}+4 g_{3}^{2} g_{2}^{2}+28 \lambda_{\phi}^{\prime \prime 2}+\frac{1}{2} \kappa_{H \phi}^{\prime 2}+24 \lambda_{\phi} \lambda_{\phi}^{\prime \prime}+\frac{32}{3} \lambda_{\phi}^{\prime} \lambda_{\phi}^{\prime \prime}\right),
\end{aligned}
$$

(vii) $\left(\mathbf{6}^{*}, \mathbf{1}, Y_{\phi}\right), N_{f}=1$

$$
\begin{aligned}
\frac{d \lambda_{\phi}}{d t} & =\frac{1}{16 \pi^{2}}\left(-40 g_{3}^{2} \lambda_{\phi}-12 \lambda_{\phi} g_{Y}^{2} Y_{\phi}^{2}+\frac{160}{9} \lambda^{\prime 2}{ }_{\phi}+\frac{80}{3} \lambda_{\phi} \lambda_{\phi}^{\prime}+40 \lambda_{\phi}^{2}+\frac{\kappa_{\phi X}^{2}}{2}+6 g_{Y}^{4} Y_{\phi}^{4}+\frac{40 g_{3}^{4}}{3}+2 \kappa_{H \phi}^{2}\right), \\
\frac{d \lambda_{\phi}^{\prime}}{d t} & =\frac{1}{16 \pi^{2}}\left(-40 g_{3}^{2} \lambda_{\phi}^{\prime}-12 g_{Y}^{2} Y_{\phi}^{2} \lambda_{\phi}^{\prime}+28 \lambda^{\prime 2}{ }_{\phi}+24 \lambda_{\phi} \lambda_{\phi}^{\prime}+12 g_{3}^{2} g_{Y}^{2} Y_{\phi}^{2}+\frac{5 g_{3}^{4}}{2}\right),
\end{aligned}
$$

(viii) $\left(\mathbf{6}^{*}, \mathbf{3}, Y_{\phi}\right), N_{f}=1^{19}$

$$
\begin{aligned}
& \frac{d \lambda_{\mathrm{tr}}}{d t}=\frac{1}{16 \pi^{2}}\left(-32 \lambda^{\prime \prime \prime}{ }_{\phi} \lambda_{\mathrm{tr}}+16 \lambda_{\mathrm{tr}} \lambda_{\phi}^{\prime}+24 \lambda_{\phi} \lambda_{\mathrm{tr}}+36 \lambda_{\mathrm{tr}}^{2}-24 g_{2}^{2} \lambda_{\mathrm{tr}}-40 g_{3}^{2} \lambda_{\mathrm{tr}}-12 g_{Y}^{2} \lambda_{\mathrm{tr}} Y_{\phi}^{2}+6 g_{2}^{4}+6 g_{3}^{4}\right. \\
& \left.+8 \lambda_{\phi}^{\prime 2}+8 \lambda_{\phi}^{\prime \prime 2}+\frac{176}{9} \lambda_{\phi}^{\prime \prime \prime 2}-16 \lambda_{\phi}^{\prime} \lambda_{\phi}^{\prime \prime \prime}-\frac{32}{3} \lambda_{\phi}^{\prime \prime} \lambda_{\phi}^{\prime \prime \prime}\right) \\
& \frac{d \lambda_{\phi}^{\prime \prime \prime}}{d t}=\frac{1}{16 \pi^{2}}\left(36 \lambda^{\prime \prime \prime 2}{ }_{\phi}+24 \lambda_{\phi} \lambda^{\prime \prime \prime}{ }_{\phi}+\frac{64}{3} \lambda^{\prime \prime \prime}{ }_{\phi} \lambda_{\phi}^{\prime}+16 \lambda_{\phi}^{\prime} \lambda_{\phi}^{\prime \prime}+8 \lambda^{\prime \prime \prime}{ }_{\phi} \lambda_{\text {tr }}-24 g_{2}^{2} \lambda^{\prime \prime \prime}{ }_{\phi}-40 g_{3}^{2} \lambda^{\prime \prime \prime}{ }_{\phi}-12 g_{Y}^{2} \lambda^{\prime \prime \prime}{ }_{\phi} Y_{\phi}^{2}+12 g_{3}^{2} g_{2}^{2}\right) \text {, } \\
& \frac{d \lambda_{\phi}^{\prime \prime}}{d t}=\frac{1}{16 \pi^{2}}\left(-\frac{80}{9} \lambda^{\prime \prime \prime 2}{ }_{\phi}+52 \lambda^{\prime \prime 2}{ }_{\phi}+24 \lambda_{\phi} \lambda_{\phi}^{\prime \prime}+\frac{80}{3} \lambda^{\prime \prime \prime}{ }_{\phi} \lambda_{\phi}^{\prime \prime}+\frac{320}{9} \lambda^{\prime \prime \prime}{ }_{\phi} \lambda_{\phi}^{\prime}+\frac{80}{3} \lambda_{\phi}^{\prime} \lambda_{\phi}^{\prime \prime}\right. \\
& \left.+\frac{80}{3} \lambda^{\prime \prime \prime}{ }_{\phi} \lambda_{\mathrm{tr}}+64 \lambda_{\mathrm{tr}} \lambda_{\phi}^{\prime \prime}-24 g_{2}^{2} \lambda_{\phi}^{\prime \prime}-40 g_{3}^{2} \lambda_{\phi}^{\prime \prime}-12 g_{Y}^{2} Y_{\phi}^{2} \lambda_{\phi}^{\prime \prime}+12 g_{2}^{2} g_{Y}^{2} Y_{\phi}^{2}-3 g_{2}^{4}+\frac{1}{2} \kappa_{\mathrm{H} \phi}^{\prime 2}\right), \\
& \frac{d \lambda_{\phi}^{\prime}}{d t}=\frac{1}{16 \pi^{2}}\left(\frac{20}{3} \lambda^{\prime \prime \prime 2}{ }_{\phi}+48 \lambda^{\prime 2}{ }_{\phi}+24 \lambda_{\phi} \lambda_{\phi}^{\prime}+\frac{88}{3} \lambda^{\prime \prime \prime}{ }_{\phi} \lambda_{\phi}^{\prime}+16 \lambda_{\phi}^{\prime} \lambda_{\phi}^{\prime \prime}+32 \lambda_{\phi}^{\prime \prime \prime} \lambda_{\phi}^{\prime \prime \prime}+16 \lambda^{\prime \prime \prime}{ }_{\phi} \lambda_{\mathrm{tr}}+40 \lambda_{\mathrm{tr}} \lambda_{\phi}^{\prime}\right. \\
& \left.-40 g_{3}^{2} \lambda_{\phi}^{\prime}-24 g_{2}^{2} \lambda_{\phi}^{\prime}-12 g_{Y}^{2} Y_{\phi}^{2} \lambda_{\phi}^{\prime}+12 g_{3}^{2} g_{Y}^{2} Y_{\phi}^{2}+\frac{5 g_{3}^{4}}{2}\right), \\
& \frac{d \lambda_{\phi}}{d t}=\frac{1}{16 \pi^{2}}\left(16 \lambda^{\prime \prime \prime}{ }_{\phi}+\frac{160}{3} \lambda_{\phi} \lambda^{\prime \prime \prime}{ }_{\phi}+8 \lambda^{\prime \prime 2}{ }_{\phi}+16 \lambda_{\phi} \lambda_{\phi}^{\prime \prime}+\frac{88}{9} \lambda_{\phi}^{\prime 2}+\frac{80}{3} \lambda_{\phi} \lambda_{\phi}^{\prime}+\frac{32}{3} \lambda^{\prime \prime \prime}{ }_{\phi} \lambda_{\phi}^{\prime \prime}+16 \lambda^{\prime \prime \prime}{ }_{\phi} \lambda_{\phi}^{\prime}+88 \lambda_{\phi}^{2}+32 \lambda^{\prime \prime \prime}{ }_{\phi} \lambda_{\text {tr }}\right. \\
& \left.+16 \lambda_{\mathrm{tr}} \lambda_{\phi}^{\prime \prime}+\frac{32}{3} \lambda_{\mathrm{tr}} \lambda_{\phi}^{\prime}+72 \lambda_{\phi} \lambda_{\mathrm{tr}}+12 \lambda_{\mathrm{tr}}^{2}-24 g_{2}^{2} \lambda_{\phi}-40 g_{3}^{2} \lambda_{\phi}-12 g_{Y}^{2} \lambda_{\phi} Y_{\phi}^{2}+6 g_{Y}^{4} Y_{\phi}^{4}+6 g_{2}^{4}+\frac{22 g_{3}^{4}}{3}+2 \kappa_{\mathrm{H} \phi}^{2}+\frac{\kappa_{\phi X}^{2}}{2}\right),
\end{aligned}
$$

\footnotetext{
${ }_{18}^{18} \lambda_{\text {ad }}$ is not induced at one loop level.

${ }^{19} \lambda_{\mathrm{ad}}$ is not induced at one loop level.
} 
(ix) $\left(\mathbf{8}, \mathbf{2}, Y_{\phi}\right), N_{f}=1$

$$
\begin{aligned}
\frac{d \lambda_{\phi}}{d t}= & \frac{1}{16 \pi^{2}}\left(-36 g_{3}^{2} \lambda_{\phi}-9 g_{2}^{2} \lambda_{\phi}-12 Y_{\phi}^{2} g_{Y}^{2} \lambda_{\phi}+\frac{9}{8} g_{2}^{4}+\frac{27}{4} g_{3}^{4}+6 g_{Y}^{4} Y_{\phi}^{4}+2 \kappa_{H \phi}^{2}+\frac{1}{2} \kappa_{\phi X}^{2}\right. \\
& \left.+80 \lambda_{\phi}^{2}+9 \lambda_{\phi}^{\prime 2}+\frac{3}{2} \lambda_{\phi}^{\prime \prime 2}+6 \lambda_{\phi} \lambda_{\phi}^{\prime \prime}+18 \lambda_{\phi} \lambda_{\phi}^{\prime \prime \prime}+\frac{9}{2} \lambda_{\phi}^{\prime} \lambda_{\phi}^{\prime \prime \prime}+\frac{27}{16} \lambda_{\phi}^{\prime \prime \prime 2}+24 \lambda_{\phi} \lambda_{\phi}^{\prime}+12 \lambda_{\mathrm{ad}}^{\prime 2}+24 \lambda_{\mathrm{ad}}^{\prime} \lambda_{\phi}\right), \\
\frac{d \lambda_{\phi}^{\prime}}{d t}= & \frac{1}{16 \pi^{2}}\left(-36 g_{3}^{2} \lambda_{\phi}^{\prime}-9 g_{2}^{2} \lambda_{\phi}^{\prime}-12 Y_{\phi}^{2} g_{Y}^{2} \lambda_{\phi}^{\prime}+12 Y_{\phi}^{2} g_{Y}^{2} g_{3}^{2}+36 \lambda_{\phi}^{\prime 2}+24 \lambda_{\phi} \lambda_{\phi}^{\prime}+6 \lambda_{\phi}^{\prime} \lambda_{\phi}^{\prime \prime}\right. \\
& \left.+3 \lambda_{\phi}^{\prime \prime} \lambda_{\phi}^{\prime \prime \prime}+12 \lambda_{\phi}^{\prime} \lambda_{\phi}^{\prime \prime \prime}-24 \lambda_{\mathrm{ad}}^{\prime} \lambda_{\phi}^{\prime}-6 \lambda_{\mathrm{ad}}^{\prime} \lambda_{\phi}^{\prime \prime \prime}\right), \\
\frac{d \lambda_{\phi}^{\prime \prime}}{d t}= & \frac{1}{16 \pi^{2}}\left(-36 g_{3}^{2} \lambda_{\phi}^{\prime \prime}-9 g_{2}^{2} \lambda_{\phi}^{\prime \prime}-12 Y_{\phi}^{2} g_{Y}^{2} \lambda_{\phi}^{\prime \prime}+9 g_{3}^{4}+12 Y_{\phi}^{2} g_{Y}^{2} g_{2}^{2}+\frac{1}{2}\left(\kappa_{H \phi}^{\prime}\right)^{2}+14 \lambda_{\phi}^{\prime \prime 2}+12 \lambda_{\phi}^{\prime 2}\right. \\
& \left.+24 \lambda_{\phi} \lambda_{\phi}^{\prime \prime}+24 \lambda_{\phi}^{\prime} \lambda_{\phi}^{\prime \prime}-6 \lambda_{\phi}^{\prime \prime} \lambda_{\phi}^{\prime \prime \prime}+6 \lambda_{\phi}^{\prime} \lambda_{\phi}^{\prime \prime \prime}+\frac{9}{4} \lambda_{\phi}^{\prime \prime \prime 2}+32 \lambda_{\mathrm{ad}}^{\prime 2}+8 \lambda_{\mathrm{ad}}^{\prime} \lambda_{\phi}^{\prime \prime}\right), \\
\frac{d \lambda_{\phi}^{\prime \prime \prime}}{d t}= & \frac{1}{16 \pi^{2}}\left(-36 g_{3}^{2} \lambda_{\phi}^{\prime \prime \prime}-9 g_{2}^{2} \lambda_{\phi}^{\prime \prime \prime}-12 Y_{\phi}^{2} g_{Y}^{2} \lambda_{\phi}^{\prime \prime \prime}+6 g_{3}^{4}+12 g_{2}^{2} g_{3}^{2}+8 \lambda_{\phi}^{\prime 2}+24 \lambda_{\phi} \lambda_{\phi}^{\prime \prime \prime}+16 \lambda_{\phi}^{\prime} \lambda_{\phi}^{\prime \prime}\right. \\
& \left.+4 \lambda_{\phi}^{\prime} \lambda_{\phi}^{\prime \prime \prime}+\frac{15}{2} \lambda_{\phi}^{\prime \prime \prime 2}-2 \lambda_{\phi}^{\prime \prime} \lambda_{\phi}^{\prime \prime \prime}+16 \lambda_{\mathrm{ad}} \lambda_{\phi}^{\prime \prime \prime}\right), \\
\frac{d \lambda_{\mathrm{ad}}^{\prime}}{d t}= & \frac{1}{16 \pi^{2}}\left(-36 g_{3}^{2} \lambda_{\mathrm{ad}}^{\prime}-9 g_{2}^{2} \lambda_{\mathrm{ad}}^{\prime}+\frac{9 g_{3}^{4}}{2}-12 \lambda_{\mathrm{ad}}^{\prime} g_{Y}^{2} Y_{\phi}^{2}+\frac{9}{8} \lambda^{\prime \prime \prime 2}{ }_{\phi}-6 \lambda_{\mathrm{ad}}^{\prime} \lambda_{\phi}^{\prime \prime \prime}+10 \lambda_{\mathrm{ad}}^{\prime} \lambda_{\phi}^{\prime \prime}+6 \lambda_{\phi}^{\prime 2}-24 \lambda_{\mathrm{ad}}^{\prime} \lambda_{\phi}^{\prime}\right. \\
& \left.+3 \lambda^{\prime \prime \prime}{ }_{\phi} \lambda_{\phi}^{\prime}+24 \lambda_{\mathrm{ad}}^{\prime} \lambda_{\phi}+32 \lambda_{\mathrm{ad}}^{2}\right) .
\end{aligned}
$$

[1] C. D. Froggatt and H. B. Nielsen, Phys. Lett. B 368, 96 (1996); M. Shaposhnikov and C. Wetterich, Phys. Lett. B 683, 196 (2010).

[2] M. Holthausen, K. S. Lim, and M. Lindner, J. High Energy Phys. 02 (2012) 037; F. Bezrukov, M. Y. Kalmykov, B. A. Kniehl, and M. Shaposhnikov, J. High Energy Phys. 10 (2012) 140; Y. Hamada, H. Kawai, and K. y. Oda, Phys. Rev. D 87, 053009 (2013); 89, 059901 (2014); F. Jegerlehner, Acta Phys. Pol. B 45, 1167 (2014); arXiv: 1305.6652; Y. Hamada, H. Kawai, and K. y. Oda, arXiv: 1305.7055; D. Buttazzo, G. Degrassi, P. P. Giardino, G. F. Giudice, F. Sala, A. Salvio, and A. Strumia, J. High Energy Phys. 12 (2013) 089; V. Branchina and E. Messina, Phys. Rev. Lett. 111, 241801 (2013); Y. Kawamura, Prog. Theor. Exp. Phys. (2013) 113B04; W. Chao, M. Gonderinger, and M. J. Ramsey-Musolf, Phys. Rev. D 86, 113017 (2012); A. Kobakhidze and A. Spencer-Smith, arXiv:1404.4709; N. Khan and S. Rakshit, Phys. Rev. D 90, 113008 (2014); 92, 055006 (2015); N. Haba, H. Ishida, K. Kaneta, and R. Takahashi, Phys. Rev. D 90, 036006 (2014); Y. Abe, M. Horikoshi, and T. Inami, arXiv:1602.03792; F. L. Bezrukov and M. Shaposhnikov, Phys. Lett. B 659, 703 (2008); K. Allison, J. High Energy Phys. 02 (2014) 040. Y. Hamada, H. Kawai, and K. y. Oda, Prog. Theor. Exp. Phys. (2014)
023B02; F. Jegerlehner, Acta Phys. Pol. B 45, 1215 (2014); Y. Hamada, H. Kawai, K. y. Oda, and S. C. Park, Phys. Rev. Lett. 112, 241301 (2014); F. Bezrukov and M. Shaposhnikov, Phys. Lett. B 734, 249 (2014); N. Haba and R. Takahashi, Phys. Rev. D 89, 115009 (2014); 90, 039905 (2014); Y. Hamada, H. Kawai, and K. y. Oda, J. High Energy Phys. 07 (2014) 026; P. Ko and W. I. Park, arXiv:1405.1635; N. Haba, H. Ishida, and R. Takahashi, Prog. Theor. Exp. Phys. (2015) 053B01; H. J. He and Z. Z. Xianyu, J. Cosmol. Astropart. Phys. 10 (2014) 019; Y. Hamada, H. Kawai, K. y. Oda, and S. C. Park, Phys. Rev. D 91, 053008 (2015); Y. Hamada, K. y. Oda, and F. Takahashi, Phys. Rev. D 90, 097301 (2014); Y. Hamada, H. Kawai, and K. y. Oda, Phys. Rev. D 92, 045009 (2015); N. Okada and Q. Shafi, Phys. Lett. B 747, 223 (2015); I. D. Saltas, arXiv:1512.06134; T. Inagaki, R. Nakanishi, and S. D. Odintsov, Phys. Lett. B 745, 105 (2015); F. Jegerlehner, arXiv:1503.00809; Y. Abe, T. Inami, Y. Kawamura, and Y. Koyama, Prog. Theor. Exp. Phys. (2015) 093B03; K. Bamba, S. D. Odintsov, and P. V. Tretyakov, Eur. Phys. J. C 75, 344 (2015); L. Sebastiani and R. Myrzakulov, Int. J. Geom. Methods Mod. Phys. 12, 1530003 (2015); I. D. Saltas, J. Cosmol. Astropart. Phys. 02 (2016) 048; A. Salvio and A. Mazumdar, Phys. Lett. B 750, 194 (2015). 
[3] H. Kawai and T. Okada, Prog. Theor. Phys. 127, 689 (2012); H. Kawai, Int. J. Mod. Phys. A 28, 1340001 (2013); Y. Hamada, H. Kawai, and K. Kawana, Int. J. Mod. Phys. A 29, 1450099 (2014); Prog. Theor. Exp. Phys. (2015) 033B06; K. Kawana, Prog. Theor. Exp. Phys. (2015) 023B04; Y. Hamada, H. Kawai, and K. Kawana, Prog. Theor. Exp. Phys. (2015) 091B01; (2015) 123B03.

[4] K. A. Meissner and H. Nicolai, Phys. Lett. B 648, 312 (2007); R. Foot, A. Kobakhidze, K. L. McDonald, and R. R. Volkas, Phys. Rev. D 77, 035006 (2008); K. A. Meissner and H. Nicolai, Phys. Lett. B 660, 260 (2008); S. Iso, N. Okada, and Y. Orikasa, Phys. Lett. B 676, 81 (2009); Phys. Rev. D 80, 115007 (2009); L. Alexander-Nunneley and A. Pilaftsis, J. High Energy Phys. 09 (2010) 021; S. Iso and Y. Orikasa, Prog. Theor. Exp. Phys. (2013) 023B08; R. Foot, A. Kobakhidze, and A. Spencer-Smith, Phys. Lett. B 747, 169 (2015); H. Okada and Y. Orikasa, arXiv: 1412.3616; K. Kawana, Prog. Theor. Exp. Phys. (2015) 073B04; I. Oda, Adv. Stud. Theor. Phys. 9, 595 (2015); N. Haba and Y. Yamaguchi, Prog. Theor. Exp. Phys. (2015) $093 \mathrm{~B} 05$.

[5] The ATLAS Collaboration, Report No. ATLAS-CONF2015-081.

[6] CMS Collaboration, Report No. CMS-PAS-EXO-15-004.

[7] L. D. Landau, Dokl. Akad. Nauk Ser. Fiz. 60, 207 (1948).

[8] C. N. Yang, Phys. Rev. 77, 242 (1950).

[9] K. Harigaya and Y. Nomura, Phys. Lett. B 754, 151 (2016); Y. Mambrini, G. Arcadi, and A. Djouadi, Phys. Lett. B 755, 426 (2016); M. Backovic, A. Mariotti, and D. Redigolo, J. High Energy Phys. 03 (2016) 157; A. Angelescu, A. Djouadi, and G. Moreau, Phys. Lett. B 756, 126 (2016); Y. Nakai, R. Sato, and K. Tobioka, Phys. Rev. Lett. 116, 151802 (2016); D. Buttazzo, A. Greljo, and D. Marzocca, Eur. Phys. J. C 76, 116 (2016); A. Pilaftsis, Phys. Rev. D 93, 015017 (2016); R. Franceschini, G. F. Giudice, J. F. Kamenik, M. McCullough, A. Pomarol, R. Rattazzi, M. Redi, F. Riva, A. Strumia, and R. Torre, J. High Energy Phys. 03 (2016) 144; S. Di Chiara, L. Marzola, and M. Raidal, Phys. Rev. D 93, 095018 (2016); T. Higaki, K. S. Jeong, N. Kitajima, and F. Takahashi, Phys. Lett. B 755, 13 (2016); S. D. McDermott, P. Meade, and H. Ramani, Phys. Lett. B 755, 353 (2016); M. Low, A. Tesi, and L. T. Wang, J. High Energy Phys. 03 (2016) 108; B. Bellazzini, R. Franceschini, F. Sala, and J. Serra, J. High Energy Phys. 04 (2016) 072; R. S. Gupta, S. Jäger, Y. Kats, G. Perez, and E. Stamou, arXiv:1512.05332; C. Petersson and R. Torre, Phys. Rev. Lett. 116, 151804 (2016); E. Molinaro, F. Sannino, and N. Vignaroli, arXiv:1512.05334; B. Dutta, Y. Gao, T. Ghosh, I. Gogoladze, and T. Li, Phys. Rev. D 93, 055032 (2016); Q. H. Cao, Y. Liu, K.P. Xie, B. Yan, and D. M. Zhang, arXiv:1512.05542; S. Matsuzaki and K. Yamawaki, arXiv:1512.05564; A. Kobakhidze, F. Wang, L. Wu, J. M. Yang, and M. Zhang, Phys. Lett. B 757, 92 (2016); R. Martinez, F. Ochoa, and C. F. Sierra, arXiv: 1512.05617; P. Cox, A. D. Medina, T. S. Ray, and A. Spray, arXiv:1512.05618; J. M. No, V. Sanz, and J. Setford, Phys. Rev. D 93, 095010 (2016); S. V. Demidov and D. S. Gorbunov, JETP Lett. 103, 219 (2016); W. Chao, R. Huo, and J. H. Yu, arXiv:1512.05738; S. Fichet, G. von
Gersdorff, and C. Royon, arXiv:1512.05751; D. Curtin and C. B. Verhaaren, Phys. Rev. D 93, 055011 (2016); L. Bian, N. Chen, D. Liu, and J. Shu, Phys. Rev. D 93, 095011 (2016); J. Chakrabortty, A. Choudhury, P. Ghosh, S. Mondal, and T. Srivastava, arXiv:1512.05767; A. Ahmed, B. M. Dillon, B. Grzadkowski, J. F. Gunion, and Y. Jiang, arXiv:1512.05771; P. Agrawal, J. Fan, B. Heidenreich, M. Reece, and M. Strassler, arXiv:1512.05775; C. Csaki, J. Hubisz, and J. Terning, arXiv:1512.05776; A. Falkowski, O. Slone, and T. Volansky, J. High Energy Phys. 02 (2016) 152; D. Aloni, K. Blum, A. Dery, A. Efrati, and Y. Nir, arXiv:1512.05778; Y. Bai, J. Berger, and R. Lu, Phys. Rev. D 93, 076009 (2016); E. Gabrielli, K. Kannike, B. Mele, M. Raidal, C. Spethmann, and H. Veermäe, Phys. Lett. B 756, 36 (2016); R. Benbrik, C. H. Chen, and T. Nomura, Phys. Rev. D 93, 055034 (2016); J. S. Kim, J. Reuter, K. Rolbiecki, and R. R. de Austri, Phys. Lett. B 755, 403 (2016); A. Alves, A. G. Dias, and K. Sinha, Phys. Lett. B 757, 39 (2016); E. Megias, O. Pujolas, and M. Quiros, J. High Energy Phys. 05 (2016) 137; L. M. Carpenter, R. Colburn, and J. Goodman, arXiv:1512.06107; J. Bernon and C. Smith, Phys. Lett. B 757, 148 (2016); W. Chao, Phys. Rev. D 93, 115013 (2016); M. T. Arun and P. Saha, arXiv:1512.06335; C. Han, H. M. Lee, M. Park, and V. Sanz, Phys. Lett. B 755, 371 (2016); S. Chang, Phys. Rev. D 93, 055016 (2016); I. Chakraborty and A. Kundu, Phys. Rev. D 93, 055003 (2016); R. Ding, L. Huang, T. Li, and B. Zhu, arXiv:1512.06560; H. Han, S. Wang, and S. Zheng, Nucl. Phys. B907, 180 (2016); X. F. Han and L. Wang, Phys. Rev. D 93, 055027 (2016); M. x. Luo, K. Wang, T. Xu, L. Zhang, and G. Zhu, Phys. Rev. D 93, 055042 (2016); J. Chang, K. Cheung, and C. T. Lu, Phys. Rev. D 93, 075013 (2016); D. Bardhan, D. Bhatia, A. Chakraborty, U. Maitra, S. Raychaudhuri, and T. Samui, arXiv:1512.06674; T. F. Feng, X. Q. Li, H. B. Zhang, and S. M. Zhao, arXiv:1512.06696; O. Antipin, M. Mojaza, and F. Sannino, Phys. Rev. D 93, 115007 (2016); F. Wang, L. Wu, J. M. Yang, and M. Zhang, Phys. Lett. B 759, 191 (2016); J. Cao, C. Han, L. Shang, W. Su, J. M. Yang, and Y. Zhang, Phys. Lett. B 755, 456 (2016); F. P. Huang, C. S. Li, Z. L. Liu, and Y. Wang, arXiv:1512.06732; W. Liao and H. q. Zheng, arXiv:1512.06741; J. J. Heckman, Nucl. Phys. B906, 231 (2016); M. Dhuria and G. Goswami, arXiv:1512.06782; X. J. Bi, Q. F. Xiang, P. F. Yin, and Z. H. Yu, Nucl. Phys. B909, 43 (2016); J. S. Kim, K. Rolbiecki, and R. R. de Austri, Eur. Phys. J. C 76, 251 (2016); L. Berthier, J. M. Cline, W. Shepherd, and M. Trott, J. High Energy Phys. 04 (2016) 084; W. S. Cho, D. Kim, K. Kong, S. H. Lim, K. T. Matchev, J. C. Park, and M. Park, Phys. Rev. Lett. 116, 151805 (2016); J. M. Cline and Z. Liu, arXiv:1512.06827; M. Bauer and M. Neubert, arXiv:1512.06828; M. Chala, M. Duerr, F. Kahlhoefer, and K. Schmidt-Hoberg, Phys. Lett. B 755, 145 (2016); D. Barducci, A. Goudelis, S. Kulkarni, and D. Sengupta, J. High Energy Phys. 05 (2016) 154; S. M. Boucenna, S. Morisi, and A. Vicente, Phys. Rev. D 93, 115008 (2016); C. W. Murphy, Phys. Lett. B 757, 192 (2016); A. E. C. Hernandez and I. Nisandzic, arXiv:1512.07165; U. K. Dey, S. Mohanty, and G. Tomar, Phys. Lett. B 756, 384 (2016); G. M. Pelaggi, A. Strumia, and E. Vigiani, J. High Energy 
Phys. 03 (2016) 025; J. de Blas, J. Santiago, and R. VegaMorales, Phys. Lett. B 759, 247 (2016); A. Belyaev, G. Cacciapaglia, H. Cai, T. Flacke, A. Parolini, and H. Serodio, arXiv:1512.07242; P. S. B. Dev and D. Teresi, arXiv:1512.07243; W. C. Huang, Y. L. S. Tsai, and T. C. Yuan, Nucl. Phys. B909, 122 (2016); S. Moretti and K. Yagyu, Phys. Rev. D 93, 055043 (2016); K. M. Patel and P. Sharma, Phys. Lett. B 757, 282 (2016); M. Badziak, arXiv:1512.07497; S. Chakraborty, A. Chakraborty, and S. Raychaudhuri, arXiv:1512.07527; M. Badziak, arXiv:1512.07541; W. Altmannshofer, J. Galloway, S. Gori, A. L. Kagan, A. Martin, and J. Zupan, Phys. Rev. D 93, 095015 (2016); M. Cvetič, J. Halverson, and P. Langacker, arXiv:1512.07622; J. Gu and Z. Liu, Phys. Rev. D 93, 075006 (2016); B. C. Allanach, P. S. B. Dev, S. A. Renner, and K. Sakurai, arXiv:1512.07645; H. Davoudiasl and C. Zhang, Phys. Rev. D 93, 055006 (2016); N. Craig, P. Draper, C. Kilic, and S. Thomas, arXiv:1512.07733; K. Das and S. K. Rai, Phys. Rev. D 93, 095007 (2016); K. Cheung, P. Ko, J. S. Lee, J. Park, and P. Y. Tseng, arXiv:1512.07853; J. Liu, X. P. Wang, and W. Xue, arXiv:1512.07885; J. Zhang and S. Zhou, Chin. Phys. C 40, 081001 (2016); J. A. Casas, J. R. Espinosa, and J. M. Moreno, Phys. Lett. B 759, 159 (2016); L. J. Hall, K. Harigaya, and Y. Nomura, J. High Energy Phys. 03 (2016) 017; H. Han, S. Wang, and S. Zheng, arXiv:1512.07992; J. C. Park and S. C. Park, arXiv:1512.08117; A. Salvio and A. Mazumdar, Phys. Lett. B 755, 469 (2016); D. Chway, R. Dermíšek, T. H. Jung, and H. D. Kim, arXiv:1512.08221; G. Li, Y.n. Mao, Y. L. Tang, C. Zhang, Y. Zhou, and S.h. Zhu, Phys. Rev. Lett. 116, 151803 (2016); Y. L. Tang and S.h. Zhu, arXiv:1512.08323; H. An, C. Cheung, and Y. Zhang, arXiv:1512.08378; J. Cao, F. Wang, and Y. Zhang, arXiv:1512.08392; F. Wang, W. Wang, L. Wu, J. M. Yang, and M. Zhang, arXiv: 1512.08434; C. Cai, Z.H. Yu, and H. H. Zhang, Phys. Rev. D 93, 075033 (2016); Q. H. Cao, Y. Liu, K. P. Xie, B. Yan, and D. M. Zhang, Phys. Rev. D 93, 075030 (2016); J. E. Kim, Phys. Lett. B 755, 190 (2016); J. Gao, H. Zhang, and H.X. Zhu, arXiv:1512.08478; W. Chao, arXiv:1512.08484; X. J. Bi et al., arXiv:1512.08497; F. Goertz, J. F. Kamenik, A. Katz, and M. Nardecchia, J. High Energy Phys. 05 (2016) 187; L. A. Anchordoqui, I. Antoniadis, H. Goldberg, X. Huang, D. Lust, and T. R. Taylor, Phys. Lett. B 755, 312 (2016); P. S. B. Dev, R. N. Mohapatra, and Y. Zhang, J. High Energy Phys. 02 (2016) 186; N. Bizot, S. Davidson, M. Frigerio, and J.-L. Kneur, J. High Energy Phys. 03 (2016) 073; L. E. Ibanez and V. Martin-Lozano, arXiv:1512.08777; C. W. Chiang, M. Ibe, and T. T. Yanagida, J. High Energy Phys. 05 (2016) 084; S. K. Kang and J. Song, Phys. Rev. D 93, 115012 (2016); X. J. Huang, W. H. Zhang, and Y.F. Zhou, Phys. Rev. D 93, 115006 (2016); S. Kanemura, K. Nishiwaki, H. Okada, Y. Orikasa, S. C. Park, and R. Watanabe, arXiv:1512.09048; S. Kanemura, N. Machida, S. Odori, and T. Shindou, arXiv:1512.09053; P. V. Dong and N. T. K. Ngan, arXiv:1512.09073; A. E. C. Hernández, arXiv:1512.09092; Y. Jiang, Y. Y. Li, and T. Liu, Phys. Lett. B 759, 354 (2016); K. Kaneta, S. Kang, and H. S. Lee, arXiv:1512.09129; L. Marzola, A. Racioppi,
M. Raidal, F. R. Urban, and H. Veermäe, J. High Energy Phys. 03 (2016) 190; E. Ma, arXiv:1512.09159; A. Dasgupta, M. Mitra, and D. Borah, arXiv:1512.09202; S. Jung, J. Song, and Y. W. Yoon, J. High Energy Phys. 05 (2016) 009; C. T. Potter, arXiv:1601.00240; E. Palti, Nucl. Phys. B907, 597 (2016); T. Nomura and H. Okada, Phys. Lett. B 755, 306 (2016); X. F. Han, L. Wang, L. Wu, J. M. Yang, and M. Zhang, Phys. Lett. B 756, 309 (2016); P. Ko, Y. Omura, and C. Yu, J. High Energy Phys. 04 (2016) 098; K. Ghorbani and H. Ghorbani, arXiv:1601.00602; U. Danielsson, R. Enberg, G. Ingelman, and T. Mandal, arXiv:1601.00624; W. Chao, arXiv:1601.00633; C. Csaki, J. Hubisz, S. Lombardo, and J. Terning, Phys. Rev. D 93, 095020 (2016); A. Karozas, S. F. King, G. K. Leontaris, and A. K. Meadowcroft, Phys. Lett. B 757, 73 (2016); A. E. C. Hernández, I. d. M. Varzielas, and E. Schumacher, arXiv:1601.00661; T. Modak, S. Sadhukhan, and R. Srivastava, Phys. Lett. B 756, 405 (2016); B. Dutta, Y. Gao, T. Ghosh, I. Gogoladze, T. Li, Q. Shafi, and J. W. Walker, arXiv:1601.00866; F. F. Deppisch, C. Hati, S. Patra, P. Pritimita, and U. Sarkar, Phys. Lett. B 757, 223 (2016); H. Ito, T. Moroi, and Y. Takaesu, Phys. Lett. B 756, 147 (2016); H. Zhang, arXiv:1601.01355; A. Berlin, Phys. Rev. D 93, 055015 (2016); S. Bhattacharya, S. Patra, N. Sahoo, and N. Sahu, J. Cosmol. Astropart. Phys. 06 (2016) 010; F. D’Eramo, J. de Vries, and P. Panci, J. High Energy Phys. 05 (2016) 089; I. Sahin, arXiv:1601.01676; S. Fichet, G. von Gersdorff, and C. Royon, Phys. Rev. Lett. 116, 231801 (2016); D. Borah, S. Patra, and S. Sahoo, arXiv:1601.01828; D. Stolarski and R. Vega-Morales, Phys. Rev. D 93, 055008 (2016); C. Hati, Phys. Rev. D 93, 075002 (2016); P. Ko and T. Nomura, Phys. Lett. B 758, 205 (2016); J. Cao, L. Shang, W. Su, Y. Zhang, and J. Zhu, Eur. Phys. J. C 76, 239 (2016); R. Ding, Z. L. Han, Y. Liao, and X. D. Ma, Eur. Phys. J. C 76, 204 (2016); S. Alexander and L. Smolin, arXiv:1601.03091; J. H. Davis, M. Fairbairn, J. Heal, and P. Tunney, arXiv: 1601.03153; L. V. Laperashvili, H. B. Nielsen, and C. R. Das, Int. J. Mod. Phys. A 31, 1650029 (2016); I. Dorsner, S. Fajfer, and N. Kosnik, arXiv:1601.03267; A. E. Faraggi and J. Rizos, Eur. Phys. J. C 76, 170 (2016); A. Djouadi, J. Ellis, R. Godbole, and J. Quevillon, J. High Energy Phys. 03 (2016) 205; A. Ghoshal, arXiv:1601.04291; T. Nomura and H. Okada, arXiv:1601.04516; W. Chao, arXiv: 1601.04678; M. R. Buckley, arXiv:1601.04751; X. F. Han, L. Wang, and J. M. Yang, Phys. Lett. B 757, 537 (2016); H. Okada and K. Yagyu, Phys. Lett. B 756, 337 (2016); A. Martini, K. Mawatari, and D. Sengupta, Phys. Rev. D 93, 075011 (2016); Q. H. Cao, Y. Q. Gong, X. Wang, B. Yan, and L. L. Yang, Phys. Rev. D 93, 075034 (2016); C. W. Chiang and A. L. Kuo, arXiv:1601.06394; U. Aydemir and T. Mandal, arXiv:1601.06761; S. Abel and V. V. Khoze, J. High Energy Phys. 05 (2016) 063; L. A. Harland-Lang, V. A. Khoze, and M. G. Ryskin, J. High Energy Phys. 03 (2016) 182; S. F. King and R. Nevzorov, J. High Energy Phys. 03 (2016) 139; B. J. Kavanagh, arXiv:1601.07330; T. Nomura and H. Okada, Phys. Lett. B 756, 295 (2016); C. Q. Geng and D. Huang, arXiv: 1601.07385; J. Kawamura and Y. Omura, Phys. Rev. D 93, 115011 (2016); E. Bertuzzo, P. A. N. Machado, 
and M. Taoso, arXiv:1601.07508; I. Ben-Dayan and R. Brustein, arXiv:1601.07564; D. Palle, arXiv: 1601.00618; D. B. Franzosi and M. T. Frandsen, arXiv: 1601.05357; P. Anastasopoulos and M. Bianchi, arXiv: 1601.07584; A. Hektor and L. Marzola, arXiv:1602.00004; N. D. Barrie, A. Kobakhidze, M. Talia, and L. Wu, Phys. Lett. B 755, 343 (2016); L. Aparicio, A. Azatov, E. Hardy, and A. Romanino, J. High Energy Phys. 05 (2016) 077; R. Ding, Y. Fan, L. Huang, C. Li, T. Li, S. Raza, and B. Zhu, arXiv:1602.00977; K. Harigaya and Y. Nomura, J. High Energy Phys. 03 (2016) 091; T. Li, J. A. Maxin, V. E. Mayes, and D. V. Nanopoulos, arXiv:1602.01377; A. Salvio, F. Staub, A. Strumia, and A. Urbano, J. High Energy Phys. 03 (2016) 214; S. F. Ge, H. J. He, J. Ren, and Z. Z. Xianyu, Phys. Lett. B 757, 480 (2016); S. I. Godunov, A. N. Rozanov, M.I. Vysotsky, and E. V. Zhemchugov, arXiv:1602.02380; S. B. Giddings and H. Zhang, Phys. Rev. D 93, 115002 (2016); U. Ellwanger and C. Hugonie, J. High Energy Phys. 05 (2016) 114; P. Draper and D. McKeen, J. High Energy Phys. 04 (2016) 127; C. Arbelez, A. E. C. Hernández, S. Kovalenko, and I. Schmidt, arXiv:1602.03607; C. Gross, O. Lebedev, and J. M. No, arXiv:1602.03877; C. Han, T. T. Yanagida, and N. Yokozaki, Phys. Rev. D 93, 055025 (2016); B. Dasgupta, J. Kopp, and P. Schwaller, Eur. Phys. J. C 76, 277 (2016); F. Goertz, A. Katz, M. Son, and A. Urbano, arXiv:1602.04801; S. F. Mantilla, R. Martinez, F. Ochoa, and C. F. Sierra, arXiv:1602.05216; Y. J. Zhang, B. B. Zhou, and J. J. Sun, arXiv:1602.05539; F. Staub et al., arXiv:1602.05581; S. Baek and J.h. Park, Phys. Lett. B 758, 416 (2016); M. Cvetic, J. Halverson, and P. Langacker, arXiv:1602.06257; P. Ko, T. Nomura, H. Okada, and Y. Orikasa, arXiv: 1602.07214; M. Redi, A. Strumia, A. Tesi, and E. Vigiani, J. High Energy Phys. 05 (2016) 078; E. Molinaro, F. Sannino, and N. Vignaroli, arXiv:1602.07574; F. Domingo, S. Heinemeyer, J. S. Kim, and K. Rolbiecki, Eur. Phys. J. C 76, 249 (2016); J. Ren and J. H. Yu, arXiv:1602.07708; G. Lazarides and Q. Shafi, arXiv:1602.07866; C. W. Chiang, H. Fukuda, M. Ibe, and T. T. Yanagida, Phys. Rev. D 93, 095016 (2016); C. Bonilla, M. Nebot, R. Srivastava, and J. W. F. Valle, Phys. Rev. D 93, 073009 (2016); C. Han, K. Ichikawa, S. Matsumoto, M. M. Nojiri, and M. Takeuchi, J. High Energy Phys. 04 (2016) 159; B. A. Arbuzov and I. V. Zaitsev, arXiv:1602.08293; Y. Kats and M. Strassler, J. High Energy Phys. 05 (2016) 092; T. Li, J. A. Maxin, V.E. Mayes, and D. V. Nanopoulos, arXiv:1602.09099; Y. Tsai, L. T. Wang, and Y. Zhao, arXiv:1603.00024; M. He, X. G. He, and Y. Tang, Phys. Lett. B 759, 166 (2016).

[10] D. Chway, R. Dermisek, T. H. Jung, and H. D. Kim, arXiv:1512.08221.

[11] J. Ellis, S. A. R. Ellis, J. Quevillon, V. Sanz, and T. You, J. High Energy Phys. 03 (2016) 176.

[12] M. Son and A. Urbano, J. High Energy Phys. 05 (2016) 181.

[13] J. Kang, M. A. Luty, and S. Nasri, J. High Energy Phys. 09 (2008) 086.

[14] M. Cirelli, N. Fornengo, and A. Strumia, Nucl. Phys. B753, 178 (2006); M. Cirelli, A. Strumia, and M. Tamburini, Nucl. Phys. B787, 152 (2007); M. Cirelli and A. Strumia, New J. Phys. 11, 105005 (2009).
[15] K. A. Olive et al. (Particle Data Group Collaboration), Chin. Phys. C 38, 090001 (2014).

[16] S. Fichet, G. von Gersdorff, and C. Royon, Phys. Rev. D 93, 075031 (2016); C. Csaki, J. Hubisz, and J. Terning, Phys. Rev. D 93, 035002 (2016); C. Csaki, J. Hubisz, S. Lombardo, and J. Terning, Phys. Rev. D 93, 095020 (2016).

[17] M. Baak, J. Cúth, J. Haller, A. Hoecker, R. Kogler, K. Mönig, M. Schott, and J. Stelzer (Gfitter Group Collaboration), Eur. Phys. J. C 74, 3046 (2014); E. Keith and E. Ma, Phys. Rev. Lett. 79, 4318 (1997).

[18] Y. Hamada, K. Kawana, and K. Tsumura, Phys. Lett. B 747, 238 (2015).

[19] Y. Hamada, T. Noumi, S. Sun, and G. Shiu, Phys. Rev. D 93, 123514 (2016).

[20] K. J. Bae, M. Endo, K. Hamaguchi, and T. Moroi, Phys. Lett. B 757, 493 (2016).

[21] V. Khachatryan et al. (CMS Collaboration), Phys. Lett. B 747, 98 (2015).

[22] S. Calvet, B. Fuks, P. Gris, and L. Valery, J. High Energy Phys. 04 (2013) 043.

[23] G. Aad et al. (ATLAS Collaboration), Eur. Phys. J. C 73, 2263 (2013).

[24] M. Carpentier and S. Davidson, Eur. Phys. J. C 70, 1071 (2010).

[25] V. Khachatryan et al. (CMS Collaboration), Phys. Rev. D 93, 032004 (2016).

[26] V. Khachatryan et al. (CMS Collaboration), J. High Energy Phys. 07 (2015) 042.

[27] V. Khachatryan et al. (CMS Collaboration), Phys. Lett. B 739, 229 (2014).

[28] G. Aad et al. (ATLAS Collaboration), J. High Energy Phys. 09 (2014) 176.

[29] G. Aad et al. (ATLAS Collaboration), Phys. Rev. Lett. 114, 161801 (2015).

[30] CMS Collaboration, Report No. CMS-PAS-SUS-13-023.

[31] CMS Collaboration, Report No. CMS-PAS-SUS-13-018.

[32] G. Aad et al. (ATLAS Collaboration), Eur. Phys. J. C 76, 5 (2016).

[33] V. Khachatryan et al. (CMS Collaboration), Phys. Rev. D 93, 032005 (2016).

[34] S. Chatrchyan et al. (CMS Collaboration), Eur. Phys. J. C 73, 2677 (2013).

[35] S. Chatrchyan et al. (CMS Collaboration), J. High Energy Phys. 06 (2014) 055.

[36] G. Aad et al. (ATLAS Collaboration), J. High Energy Phys. 03 (2015) 041.

[37] S. Chatrchyan et al. (CMS Collaboration), Eur. Phys. J. C 72, 2189 (2012).

[38] G. Aad et al. (ATLAS Collaboration), J. High Energy Phys. 05 (2014) 071.

[39] V. Khachatryan et al. (CMS Collaboration), Eur. Phys. J. C 74, 3036 (2014).

[40] G. Aad et al. (ATLAS Collaboration), J. High Energy Phys. 10 (2014) 96.

[41] G. Abbiendi et al. (ALEPH, DELPHI, L3, OPAL and LEP Collaborations), Eur. Phys. J. C 73, 2463 (2013).

[42] G. Aad et al. (ATLAS Collaboration), J. High Energy Phys. 08 (2015) 138.

[43] S. Kanemura, M. Kikuchi, H. Yokoya, and K. Yagyu, Prog. Theor. Exp. Phys. (2015) 051B02. 
HAMADA, KAWAI, KAWANA, and TSUMURA

PHYSICAL REVIEW D 94, 014007 (2016)

[44] G. Aad et al. (ATLAS Collaboration), J. High Energy Phys. 08 (2015) 105.

[45] G. Aad et al. (ATLAS Collaboration), J. High Energy Phys. 11 (2014) 104.

[46] V. Khachatryan et al. (CMS Collaboration), Phys. Rev. D 93 (2016) 112009.

[47] V. Khachatryan et al. (CMS Collaboration), Phys. Rev. D 93, 012003 (2016).
[48] G. Aad et al. (ATLAS Collaboration), Phys. Rev. D 92, 112007 (2015).

[49] G. Aad et al. (ATLAS Collaboration), J. High Energy Phys. 09 (2015) 108.

[50] V. Khachatryan et al. (CMS Collaboration), Phys. Rev. D 90, 092007 (2014).

[51] P. Achard et al. (L3 Collaboration), Phys. Lett. B 517, 75 (2001). 